\title{
Synthesis, Spinning, and Properties of Very High Molecular Weight Poly(acrylonitrile-co-methyl acrylate) for High Performance Precursors for Carbon Fiber
}

\author{
E. Ashley Morris, ${ }^{a *}$ Matthew C. Weisenberger, ${ }^{a}$ Stephanie B. Bradley, ${ }^{\mathrm{a}}$ Mohamed \\ G. Abdallah, ${ }^{\mathrm{b}}$ Sue J. Mecham, ${ }^{\mathrm{c}}$ Priya Pisipati, ${ }^{\mathrm{c}} \mathrm{James}$ E. McGrath ${ }^{\mathrm{c}}$ \\ ${ }^{a}$ Center for Applied Energy Research, University of Kentucky, 2540 Research Park Drive, Lexington, KY, 40511, USA, \\ Ashley.morris@uky.edu \\ bOak Ridge National Laboratory, Oak Ridge, TN, USA, agm1144@yahoo.com \\ 'Department of Chemistry and the Macromolecules and Interfaces Institute, Virginia Tech, Blacksburg, VA, USA, \\ sjmecham@unc.edu
}

\begin{abstract}
In this paper, synthesis of very high molecular weight (VHMW) polyacrylonitrile-co-methyl acrylate (PAN-co-MA) polymers with weight average molecular weights of at least 1.7 million $\mathrm{g} / \mathrm{mole}$ were repeatedly achieved on a laboratory scale using emulsion polymerization. The development of a hybrid dry-jet gel solution spinning technique for the VHMW PAN-co-MA enabled continuous spinning of 100 filament count tows, 100s of meters in length. Single filaments were analyzed and tested for tensile performance. Experimentally, the hybrid spinning method coupled with VHMW polymers produced precursor fibers with excellent tensile properties, averaging $954 \mathrm{MPa}$ in strength and $15.9 \mathrm{GPa}$ in elastic modulus $(\mathrm{N}=296)$, with small filament diameters $(5 \mu \mathrm{m})$. Results indicate a strong correlation between decreasing filament diameter, facilitated by high molecular weight polymer, and exponentially increasing tensile properties, using a hybrid dry-jet gel spinning process.
\end{abstract}

\section{Keywords}

Polyacrylonitrile; PAN polymer synthesis; Gel spinning; Dry-jet spinning; Solution spinning; Molecular weight; PAN precursor; Rheology; Tensile strength; High strength carbon fiber PAN precursor

\section{Introduction}

Due to their unique properties, carbon fibers are one of the leading reinforcing fibers for lightweight, high strength and stiffness composite materials $(1,2,3)$. The quality of the precursor fiber from which the carbon fibers are derived contributes to the properties of the resultant carbon fibers $(1,3,4,5,6)$. Polyacrylonitrile (PAN) precursor derived carbon fibers dominate the market due to their good strength and modulus properties (7) and high toughness. In order to increase ease of processing and minimize cost, the molecular weights targeted are usually lower than optimal for producing the highest strength precursor fibers. Currently, most

*Corresponding author.

Email address: Ashley.morris@uky.edu

Telephone: +1 8592570373

Notice: This manuscript has been co-authored by UT-Battelle, LLC, under Contract No. DEAC05-00OR22725 with the U.S. Department of Energy. The United States Government retains and the publisher, by accepting the article for publication, acknowledges that the United States Government retains a non-exclusive, paid-up, irrevocable, world-wide license to publish or reproduce the published form of this manuscript, or allow others to do so, for United States Government purposes. 
carbon fiber is produced from acrylonitrile copolymer precursor fiber with acrylonitrile content of 95 weight $\%$ or higher and molecular weights in the range of 70,000 to $200,000 \mathrm{~g} / \mathrm{mole}(8)$. Polymer in this molecular weight range provides fibers, which have sufficient strength for textile applications, but the strength may not be optimal in precursor fibers for high performance carbon fiber. It is known that there is a strong impact of increasing molecular weight on increasing mechanical properties (9). It has been reported that very high molecular weight (VHMW), high acrylonitrile (AN) content polymers estimated at $>1,000,000 \mathrm{~g} / \mathrm{mole}$ can provide significant increases in precursor fiber strength and modulus $(10,11,12)$. This paper tests and confirms that hypothesis. VHMW polymer leads to a reduced solids content necessary to produce a spinnable dope viscosity. Therefore smaller diameter filaments can be produced using VHMW polymer. These very small diameter filaments have been shown to provide a corresponding increase in tensile strength $(7,11)$. The primary goal of this research was to investigate the development and implementation of a commercially feasible approach to synthesize and spin VHMW, high AN content (95-97 weight \%) copolymers and analyze the resulting precursor fiber tensile properties as a function of decreasing filament diameter.

\section{Experimental}

\subsection{Copolymer Synthesis}

Very high molecular weight (VHMW) copolymer was synthesized at Virginia Tech using the following procedure.

\subsubsection{Materials}

The monomers, acrylonitrile, $\geq 99+\%$ (AN) and methyl acrylate, $99 \%$ (MA), the initiator, ammonium persulfate, and the activator, sodium metabisulfite, along with magnesium sulfate used in the workup of the emulsion, were all purchased from Sigma-Aldrich. The surfactant, Dowfax 8390 (35\% in water), was kindly provided by the Dow Chemical Company. Acrylonitrile was eluted through an activated alumina column to remove the stabilizer immediately before use. Methyl acrylate was washed three times with $0.1 \mathrm{~N}$ aq sodium hydroxide to remove the stabilizer immediately before use.

\subsubsection{Copolymerization}

The free radical emulsion copolymerization of $\mathrm{AN}$ and MA was conducted in a Parr pressure reactor under a nitrogen atmosphere. A representative procedure for a 97/3 wt\% AN/MA copolymer is as follows. Deionized (DI) water $(200 \mathrm{~mL})$ was stirred and boiled for 20 minutes and then cooled without stirring immediately prior to its use in the reaction to remove dissolved oxygen. Dowfax 8390 solution (5.714 g solution, $1.7 \mathrm{wt} \%$ active surfactant) was diluted in 100 $\mathrm{mL}$ of the deoxygenated DI water at room temperature with vigorous stirring and then transferred to the Parr vessel. Acrylonitrile (97 g, $182.8 \mathrm{mmol})$ and methyl acrylate (3 g, 34.8 mmol) were weighed into beakers and each added to the Parr reactor followed by a $40 \mathrm{~mL}$ water wash with the deoxygenated DI water for each addition. The ammonium persulfate initiator $(0.212 \mathrm{~g}, 0.05 \mathrm{~mol} \%$ based on total monomers), and sodium metabisulfite activator $(0.106 \mathrm{~g}$, $0.03 \mathrm{~mol} \%$ based on total monomers) were individually weighed and dissolved in $5 \mathrm{~mL}$ of deoxygenated DI water each, and then added to the Parr vessel. The reactor head was placed securely on top of the vessel after addition of the remaining deoxygenated DI water to bring the total $\%$ solids to $25 \%$. The headspace of the Parr reactor was purged with nitrogen for 1 min and 
then sealed under a slight nitrogen pressure. The reaction temperature was automatically controlled via an internal cooling loop connected to a circulating cold water line. The reaction was stirred at $600 \mathrm{rpm}$ and maintained at $25^{\circ} \mathrm{C}$ for $24 \mathrm{~h}$. The resultant emulsion was added to $2000 \mathrm{~mL}$ of a $1 \%$ aq $\mathrm{MgSO}_{4}$ solution at $65{ }^{\circ} \mathrm{C}$ and stirred briefly to break the emulsion, then the solid polymer was filtered. The solid powder was washed by stirring in $1500 \mathrm{~mL}$ of DI water at $65{ }^{\circ} \mathrm{C}$ twice, and after the final filtration it was dried at $100{ }^{\circ} \mathrm{C}$ under vacuum overnight. The recovered yield was $89 \%$.

\subsection{Polymer Characterization}

\subsubsection{Composition Analysis by ${ }^{1} \mathrm{H}$ NMR}

${ }^{1} \mathrm{H}$ NMR in deuterated dimethylsulfoxide (DMSO-d $\mathrm{d}_{6}$ ) was used to measure AN/MA copolymer compositions using a $400 \mathrm{MHz}$ Varian NMR spectrometer. Copolymer composition was determined by comparing the integrals of the proton signals in the repeat units. The signal at 3.2 ppm (Y) corresponds to the methine $(\mathrm{CH})$ protons from AN and MA. The signal at $2.1 \mathrm{ppm}(\mathrm{X})$ corresponds to the methylene $\left(\mathrm{CH}_{2}\right)$ protons from $\mathrm{AN}$ and MA repeat units. The signal at 3.7 ppm corresponds to the methyl $\left(\mathrm{CH}_{3}\right)$ protons of the MA units. The copolymer composition was calculated from the integral values of the methylene protons using the following equations:

$$
\begin{gathered}
\text { Moles MA } \alpha\left(\frac{\int \mathrm{CH}_{3}}{3}\right) \\
(\text { Moles MA }) \times\left(86.04 \frac{g}{\text { mole }}\right)=\text { relative weight MA } \\
\text { Moles AN } \alpha\left[\left(\frac{\int \mathrm{X}}{2}\right)-\left(\frac{\int \mathrm{CH}_{3}}{3}\right)\right] \\
(\text { Moles AN }) \times\left(53.06 \frac{g}{m o l e}\right)=\text { relative weight AN } \\
\text { Weight Percent AN }=\frac{\text { relative weight AN }}{\text { relative weight AN + relative weight MA }} \times 100 \\
\text { Weight Percent MA }=100-\text { weight percent AN }
\end{gathered}
$$

\subsubsection{Molecular Weight Analysis}

Dilute solution viscosity measurements were used to analyze the relative molecular weights and estimate the viscosity average molar mass $\left(\mathrm{M}_{\mathrm{v}}\right)$ of each copolymer prepared for spinning trials. Size exclusion chromatography with multiple detectors was utilized to determine the weight average molar mass $\left(\mathrm{M}_{\mathrm{w}}\right)$ of polyacrylonitrile-co-methyl acrylate copolymers based on light scattering measurements.

\subsubsection{Inherent Viscosity in DMF}

Inherent viscosity measurements were performed using a Cannon Ubbelohde viscometer, according to ASTM D445, at $35{ }^{\circ} \mathrm{C}$ in dimethylformamide (DMF). DMF was filtered through a 
$0.45 \mu \mathrm{m}$ PTFE filter prior to preparation of the samples. Solutions with a concentration of 0.1 $\mathrm{g} / \mathrm{dL}$ were prepared by dissolving $20 \mathrm{mg}$ of the sample in $20 \mathrm{~mL}$ of pre-filtered DMF. The sample solutions were stirred at room temperature for 16-24 hours prior to testing to ensure complete dissolution. The polymer solutions were not filtered in order to avoid filtration of high molecular weight fractions. The solvent and solutions were allowed to equilibrate to $35{ }^{\circ} \mathrm{C}$ for $20 \mathrm{~min}$ in the viscometer and a total of 5 efflux times were recorded for the solvent and for the sample solutions. The inherent viscosity was calculated from the natural log of the average sample efflux time divided by the average solvent efflux time all divided by the sample concentration to provide a value in $\mathrm{dL} / \mathrm{g}$.

\subsubsection{Intrinsic Viscosity in DMF and in DMF with $0.05 \mathrm{M} \mathrm{LiBr}$}

Intrinsic viscosity measurements were performed using a Canon Ubbelohde viscometer according to ASTM D445, at $35{ }^{\circ} \mathrm{C}$ in DMF with and without $0.05 \mathrm{M} \mathrm{LiBr}$ added. DMF was filtered through a $0.2 \mu \mathrm{m}$ PTFE filter prior to preparation of the samples and the polymer solutions were not filtered before they were run. A solution of $500 \mathrm{~mL}$ of DMF with $0.05 \mathrm{M}$ $\mathrm{LiBr}$ was prepared by dissolving $2.17 \mathrm{~g}$ of $\mathrm{LiBr}$ in $500 \mathrm{~mL}$ of the prefiltered DMF and stirring at room temperature for $24 \mathrm{~h}$. Polymer solutions of concentration $0.25 \mathrm{~g} / \mathrm{dL}$ were prepared by dissolving $50 \mathrm{mg}$ of the sample in $20 \mathrm{~mL}$ of the solvent. The solutions were stirred for 1 hour at $50{ }^{\circ} \mathrm{C}$ and 1 hour at room temperature immediately prior to testing to ensure complete dissolution. Both the solvent and copolymer solutions were added to the viscometer in the temperature controlled bath and allowed to equilibrate for at least 20 minutes before efflux times were recorded. The copolymer solution was subsequently diluted inside the viscometer by addition of $4 \mathrm{~mL}$ of solvent to the original $8 \mathrm{~mL}$ of solution with manual mixing being accomplished through repeated manipulation of the solution in the viscometer with the pipette bulb. The solutions that were diluted in the viscometer were allowed to equilibrate for 15 minutes before efflux times for the new concentration were recorded. A series of six dilutions were performed in this manner, for which efflux times were recorded. The inherent and reduced viscosity was calculated for each concentration and then each was plotted versus concentration in order to determine the y-intercepts, which were evaluated, averaged, and reported as the intrinsic viscosity.

\subsubsection{Size Exclusion Chromatography (SEC) Molecular Weight Analysis}

The SEC system utilized for the analysis of the polyacrylonitrile copolymers consisted of an Agilent Infinity 1260 pump, degasser, autosampler, and column oven for managing the solvent and injecting the samples. Tosoh alpha-M columns, $10 \mu \mathrm{m}$ particle size mixed bed hydrophilic vinyl resin based columns with a broad distribution of pore sizes suitable for use with polar solvents and capable of separating polystyrene molecules up to 5,000,000 Daltons, were used for the separations. Data is also reported for alternative mixed bed columns based on polystyrene and designated as capable of separating polymers up to 2,000,000 Daltons. The detectors, from Wyatt Technologies, consisted of a DAWN Heleos II multi-angle laser light scattering (MALLS) detector, a Viscostar II viscosity detector, and a t-REX refractive index detector. The solvent system utilized was NMP, which was vacuum distilled over $\mathrm{P}_{2} \mathrm{O}_{5}$, degassed, and filtered through a $0.2 \mu \mathrm{m}$ PTFE filter before use. In cases where salt was added to the solvent, dried $\mathrm{LiBr}(0.05$ M) was added to the distilled solvent before it was degassed and filtered. Typically, SEC samples were prepared in concentrations ranging from $1.0-5.0 \mathrm{mg} / \mathrm{mL}$ as necessary to obtain good signal to noise ratio in all detectors used for analysis. The sample solutions were filtered to 
remove any dust or insoluble particles and typically a $0.22 \mu \mathrm{m}$ PTFE syringe filter was used. In cases where it was suspected that large polymer molecules may have been filtered out, a larger filter size of $1.6 \mu \mathrm{m}$ was used to minimize filtration of the sample molecules.

\subsection{Spinning of VHMW PAN-co-MA Precursor Fibers}

\subsubsection{Dope Preparation}

Spinning solutions (dopes) were prepared using a custom fabricated dope mixer, purposely-built for heating and slow stirring of the dope at controlled rates while maintaining zero-head gas space above the contained dope through means of a floating piston (analogous to a hydraulic accumulator), as shown in Figure 1. To prepare the dope, $51 \mathrm{~g}$ of VHMW polymer was handmixed with $800 \mathrm{~g}$ of Fisher Chemical reagent grade, dry $N, N$-dimethylacetamide (DMAc) to create a suspension. The suspension was placed under a rotor-stator, Silverson lab mixer (L4RT) operated at $3000 \mathrm{rpm}$ for 10 minutes with a 2 -inch diameter high shear screen in order to ensure a homogeneous mixture. The suspension was then vacuum degassed at a reduced pressure of $29.9 \mathrm{inHg}$ for 20 minutes, poured into the dope mixer, sealed with the floating piston, and subjected to a $0.25{ }^{\circ} \mathrm{C} / \mathrm{min}$ temperature ramp to $110{ }^{\circ} \mathrm{C}$ while stirring at $8 \mathrm{rpm}$. Thorough mixing of the suspension while heating ensured full dissolution of the polymer into the solvent and a homogeneous solution viscosity with minimal gel formation. Maintaining zero head gas space while mixing mitigated both the inclusion of air bubbles into the solution and the formation of a surface skin from dried polymer solution.

The fully dissolved dope was then slowly cooled to room temperature for extraction from the mixer. Dope extraction was completed using four KD Scientific $200 \mathrm{~mL}$ stainless steel syringes, fitted with Kalrez O-rings, that were filled from the bottom of the mixer through a $140 \mu \mathrm{m}$ Swagelok in-line screen filter by locking the floating piston from rising and pressurizing the internal volume with nitrogen. The open syringes were then placed in a vacuum box at 29.9 inHg for $4 \mathrm{~h}$ at room temperature to de-gas the dope. After the syringes were degassed, they were ready for integration into the dope metering pump inlet system, and sampled for rheological analysis.

\subsubsection{Rheological Analysis}

Rheological characterization of the VHMW polymer dopes was performed using a TA instruments AR-G2 parallel plate rheometer equipped with a temperature controlled Peltier plate and a 40-mm diameter upper plate geometry set at a gap of $500 \mu \mathrm{m}$. A thin layer of paraffin oil was applied to the gap edge circumference after the dope was loaded to prevent dope drying during the test. A conditioning step was completed at $25{ }^{\circ} \mathrm{C}$, with a pre-shear of $1 \mathrm{~s}^{-1}$ and equilibration of 1 minute before each step. Steady state shear viscosity analysis was completed by ramping the shear rate from 0.1 to $10 \mathrm{~s}^{-1}$ at $25^{\circ} \mathrm{C}$. Dynamic oscillatory rheological analysis was completed using a frequency sweep step from 0.1 to $500 \mathrm{rad} / \mathrm{s}$ at $25{ }^{\circ} \mathrm{C}$ with a strain amplitude of $0.01 \%$. The storage shear modulus $\left(G^{\prime}\right)$, loss shear modulus $\left(G^{\prime \prime}\right)$, complex viscosity $\left(\left|n^{*}\right|\right)$, and tan delta (phase lag angle) were analyzed as a function of angular frequency. In addition, a temperature ramp step was completed, ramping the temperature from 25 to $100{ }^{\circ} \mathrm{C}$ at $3{ }^{\circ} \mathrm{C} / \mathrm{min}$, while holding the angular frequency at $10 \mathrm{rad} / \mathrm{s}$ and the strain amplitude at $0.01 \%$. 


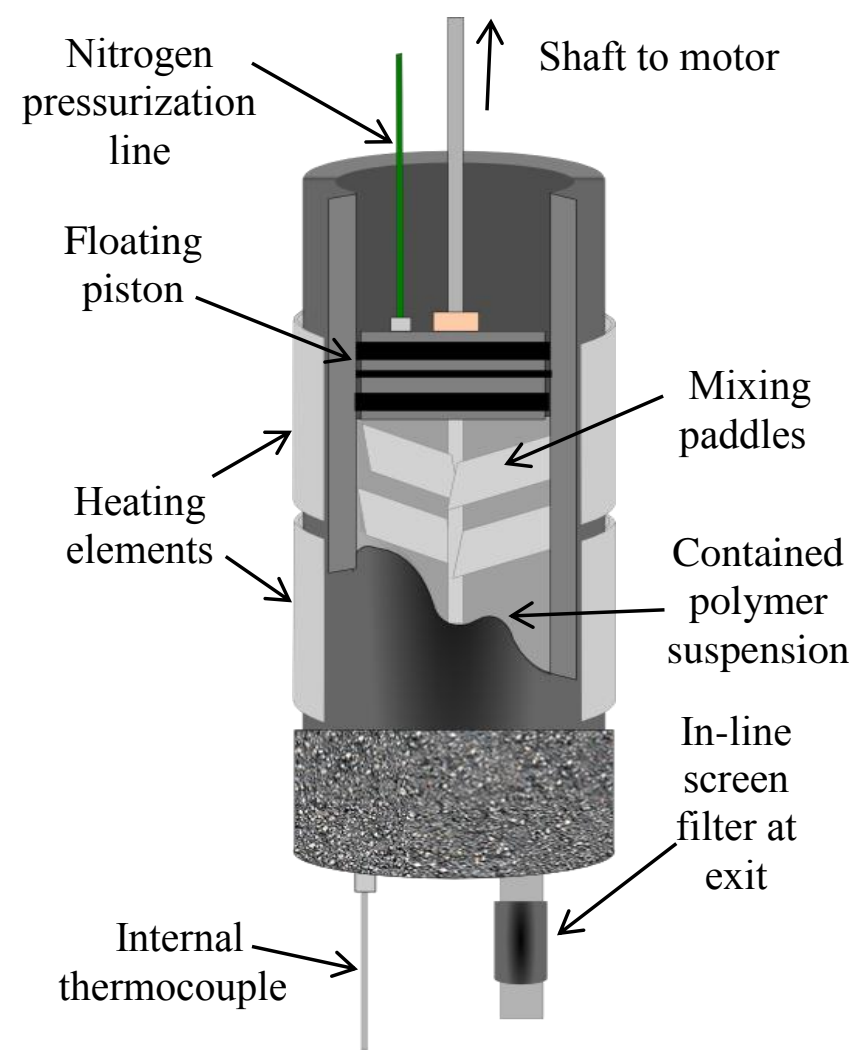

Figure 1. Schematic of the custom fabricated dope mixer for full dissolution of polymer into the solvent

\subsubsection{Fiber Spinning Equipment}

All fibers were spun utilizing the multifilament, solution fiber spinning facility at the University of Kentucky Center for Applied Energy Research. The spinning line was purposely-built to balance the production of meaningful research quantities of continuous filament tow (100-500 filament-count tow, $\sim 1 \mathrm{~km}$ of tow) from small quantities of polymer (10s to 100s of grams), while minimizing the time and effort necessary for line preparation and change-over. The line efficiently affords itself to test the spinnability of numerous experimental dopes and enables the systematic variation of a plurality of processing parameters during spinning (13).

The spinning line was fed using four 200-mL stainless steel syringes loaded with the de-gassed dopes described above, which were pressurized using pneumatic cylinders into the dope inlet system. Sintered metal in-line filters, on the outlet of each syringe, were used to pre-filter the dope before it entered the metering pump, which provided a constant volumetric flow of dope downstream through a sintered metal filter cup, for the removal of gels and other agglomerates.

At the spinnerette head, the dope was passed through a screen pack for final filtration before flowing through the breaker plate to the spinnerette capillaries, from which jets emerged to form filaments. A variation of dry-jet (or air gap) solution spinning, in which the filament jets were extruded through a small air gap prior to being drawn into the coagulation bath, was used. Dryjet spinning is used currently for producing high tenacity acrylic fibers $(7,14,15,16)$ and has several advantages over wet-jet solution spinning (where the polymer jets emerge from a spinnerette submerged in the coagulant), including: higher spin line speed, enhanced molecular 
orientation prior to coagulation (stemming from the high spin draw in the air gap), increased smoothness and luster of fiber surfaces, and discrete temperature separation between the dope and coagulation bath temperatures $(7,14,15,16)$. The spinnerette used was a 100-filament dryjet spinnerette, with $150 \mu \mathrm{m}$ capillary diameter. Figure 2 shows forming filaments passing through an air gap and entering the coagulation bath liquid surface during dry-jet spinning at the University of Kentucky Center for Applied Energy Research.

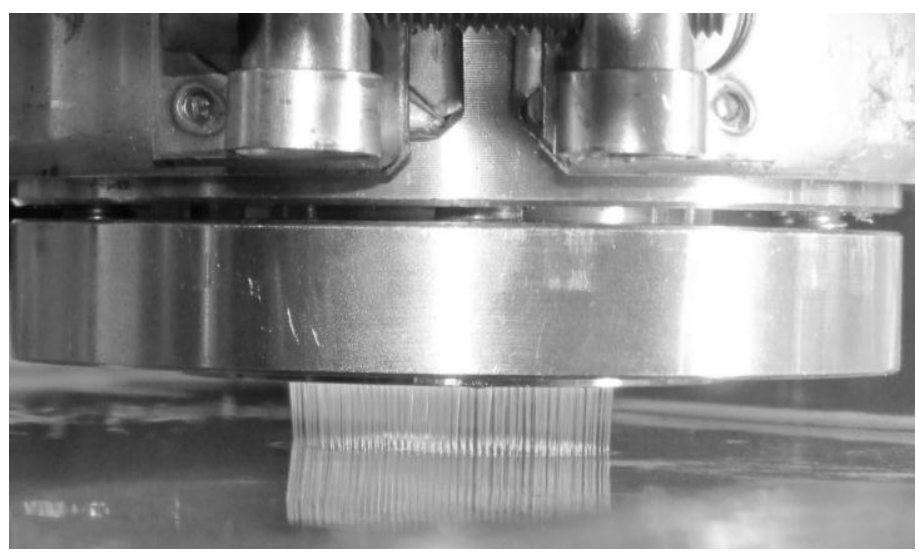

Figure 2. Nascent filament jets passing through an air gap, accelerating and attenuating from an applied spin draw, and entering the coagulation bath during dry-jet solution spinning

After passing through an air gap of 4 to $6 \mathrm{~mm}$, and entering a $60 \mathrm{wt} \%$ DMAc/ $\mathrm{H}_{2} \mathrm{O}$ coagulation bath, diffusion of solvent (DMAc) out of, and non-solvent (water) into, the forming filaments caused them to coagulate. The filaments were then passed through a sequence of wash baths, as in Figure 3. The coagulation bath was maintained near $0{ }^{\circ} \mathrm{C}$, with the temperature-controlled wash baths increasing from 5 to $30{ }^{\circ} \mathrm{C}$, followed by a hot water bath in which significant stretching occurs (to be discussed). A hot glycerol bath was used to further stretch the fiber above the polymer $\mathrm{T}_{\mathrm{g}}$, while the remaining two hot water baths provided a final level of washing prior to spin finish application, drying, and take-up using a traversing winder. The fiber tow was taken up at a rate of $56 \mathrm{~m} / \mathrm{min}$.

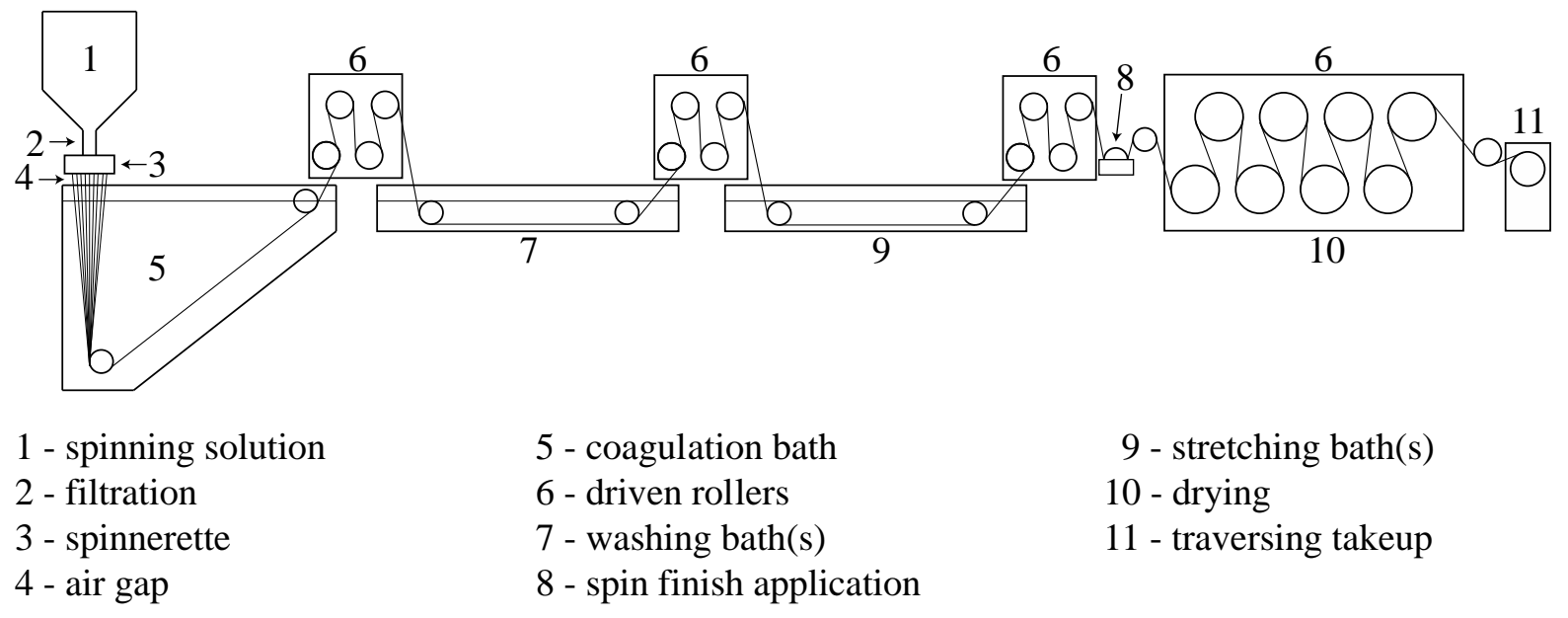

Figure 3. Schematic of solution spinning line 


\subsection{As-Spun Fiber Analysis}

\subsubsection{Optical Microscopy}

Fibers were analyzed for cross sectional shape and area using optical microscopy. Collimated fiber tows were embedded vertically into epoxy, cured, and polished for optimum resolution. Fiber cross sections were viewed through a 100x objective lens with a 10x ocular through oil using reflected light. Fiber cross section perimeters were traced by hand using SPOT Imaging Solutions Microscopy software. Cross section perimeters were converted to an equivalent circular cross section diameter. A total of 50 specimens for each sample were analyzed.

\subsubsection{Scanning Electron Microscopy (SEM)}

Imaging of the precursor fiber surface and cross sectional morphology was performed using a Hitachi S-4800 field emission SEM. Low magnification samples ( $<100 \mathrm{kx})$ were sputter-coated with gold for 120 seconds, while high magnification samples ( $\geq 100 \mathrm{kx})$ were sputter-coated with platinum for 90 seconds, both using a Hummer 6.2 Sputter System. Fiber cross-section samples were prepared for imaging by cutting the fiber bundle in liquid nitrogen using surgical scissors and placing the cut bundles vertically in the SEM sample holder. Fiber samples were prepared for surface imaging by adhering a short length of fiber bundle onto conductive carbon tape adhered to Al SEM sample holders. Fibers were imaged at $5 \mathrm{kV}$ accelerating voltage.

\subsubsection{Mechanical Measurements}

Tensile properties of precursor fiber samples were measured using approximately 20 specimens per sample at a crosshead speed of $5 \mathrm{~mm} / \mathrm{min}$. A total of 296 specimens were tested. The specimens were prepared by bonding a single filament to a $40 \mathrm{~mm}$ gauge length aperture card using Easypoxy K-20. Following curing of the epoxy, the aperture card was mounted in the miniature tensile grips of an MTS Systems Q10 machine fitted with a $150 \mathrm{~g}$ load cell. The sides of the aperture card were then cut, leaving the filament intact.

\section{Results and Discussion}

\subsection{Polymer Composition and Molecular Weight}

Twenty-four 100-g batches of polymer were synthesized and compared for estimated $\mathrm{M}_{\mathrm{v}}$ and AN content using the inherent viscosity measurements and ${ }^{1} \mathrm{H}$ NMR analyses. Figure 4 shows a representative ${ }^{1} \mathrm{H}$ NMR spectrum of a 96/4 wt\% AN/MA copolymer. Inherent viscosity measurements at $0.1 \mathrm{~g} / \mathrm{dL}$ in DMF at $35^{\circ} \mathrm{C}$ were used to estimate and compare the molar mass of polymers synthesized for spinning. It is anticipated that the inherent viscosity will be slightly higher than the intrinsic viscosity, but when comparing polymers of similar molecular weights at the same concentration, the inherent viscosity procedure provides a good measure of comparison. The viscosity average molar mass $\left(\mathrm{M}_{\mathrm{v}}\right)$ was estimated from the inherent viscosities using MarkHouwink parameters (10). The Mark-Houwink parameters are based upon the intrinsic viscosity. If the assumption is made that the solution concentration of $0.1 \mathrm{~g} / \mathrm{dL}$ is sufficiently dilute for the polymers in question to provide a reasonable estimate of the intrinsic viscosity, then the use of the Mark-Houwink parameters can provide a reasonable estimate of the $\mathrm{M}_{\mathrm{v}}$. It was noted that there was no correlation between AN content, which varied only slightly, and inherent viscosity. Although it is expected that the Mark-Houwink parameters would be affected by a change in AN 
content, the range of variation between the samples being compared is very small so the effect on the estimated $\mathrm{M}_{\mathrm{v}}$ should be minimal. Table 1 provides the statistical analysis of the copolymers used for the spinning trials. It should be noted that one copolymer was synthesized which had a measured composition of $98 \% \mathrm{AN}$, and it was found to have inherent gels in the dope that inhibited spinning of that particular material.

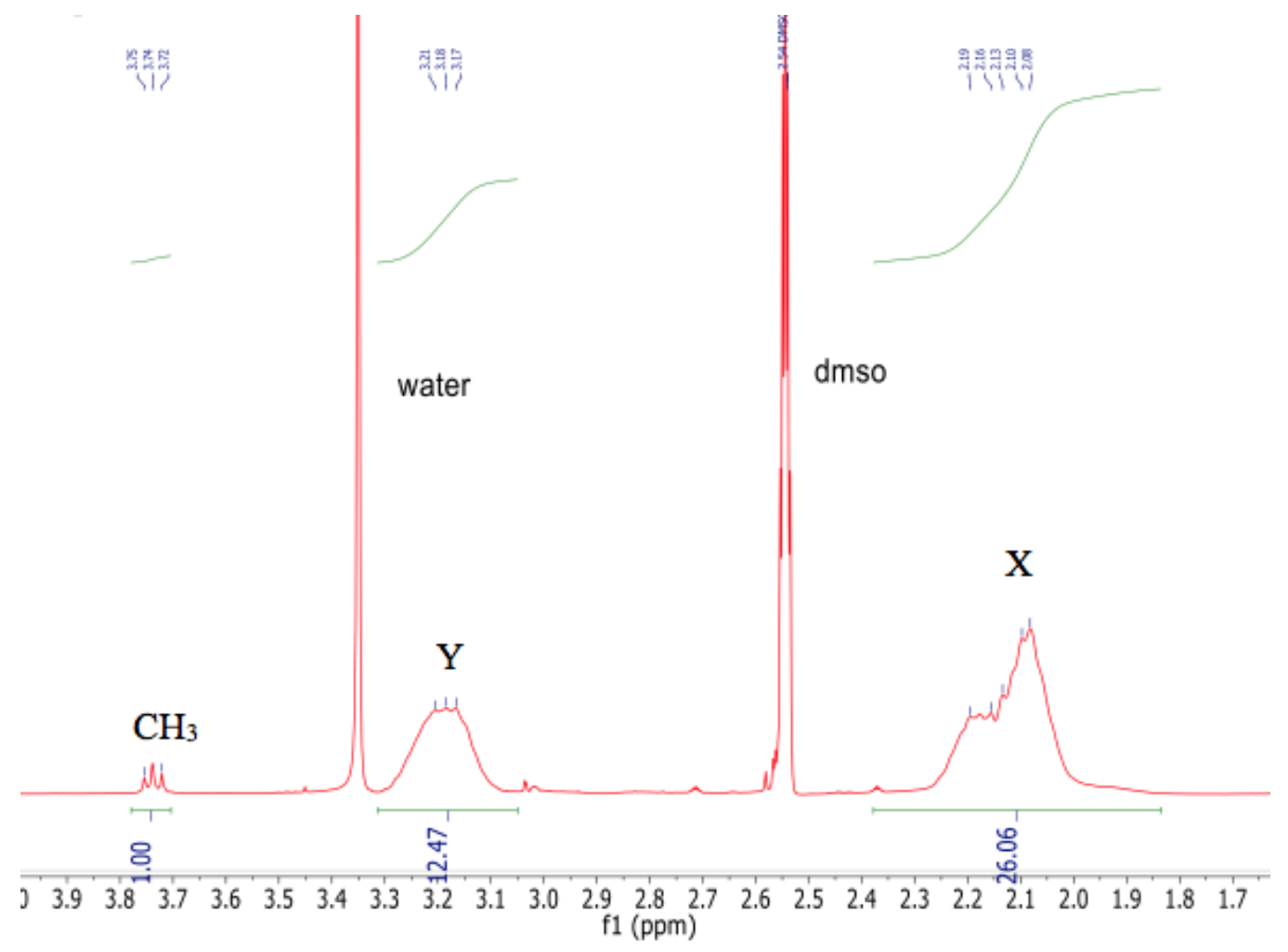

Figure $4 .{ }^{1} \mathrm{H}$ NMR of a $96 \mathrm{wt} \% \mathrm{AN}$ and $4 \mathrm{wt} \%$ MA copolymer

Table 1. Statistical analysis of 24 batches of polyacrylonitrile-co-methyl acrylate copolymers

\begin{tabular}{|c|c|c|c|}
\hline & $\begin{array}{c}\text { Acrylonitrile } \\
\text { Content (weight \%) }\end{array}$ & $\begin{array}{c}\text { Inherent Viscosity } \\
\left(0.1 \mathrm{~g} / \mathrm{dL}, \mathrm{DMF}, 35^{\circ} \mathrm{C}\right)\end{array}$ & $\begin{array}{c}\text { Estimated } \mathrm{M}_{\mathrm{v}} \\
(\mathrm{g} / \mathrm{mole})\end{array}$ \\
\hline Average & 97 & 7.8 & 775,974 \\
\hline Standard Deviation & 1 & 1.3 & 169,617 \\
\hline Count & 24 & 24 & 24 \\
\hline Low & 95 & 5.9 & 529,155 \\
\hline High & 98 & 10.9 & $1,204,817$ \\
\hline
\end{tabular}

In order to investigate the impact of measuring the inherent viscosity at $0.1 \mathrm{~g} / \mathrm{dL}$ instead of the intrinsic viscosity, a sample was tested at multiple lower concentrations, in order to determine the intrinsic viscosity for comparison. For this sample, the inherent viscosity was measured to be $10.9(\mathrm{dL} / \mathrm{g})$ and the intrinsic viscosity was $9.8(\mathrm{dL} / \mathrm{g})$, translating into calculated $\mathrm{M}_{\mathrm{v}}$ values of 1.2 and 1.04 million $\mathrm{g} / \mathrm{mole}$ respectively. It was noted, however, that the sample preparation may have had the most significant impact on this observed difference. The analogous inherent viscosity value measured during the intrinsic viscosity experiment was $9.0 \mathrm{dL} / \mathrm{g}$ in the same solvent system prepared by stirring at $50{ }^{\circ} \mathrm{C}$ for 1 hour and room temperature for 1 hour. The original inherent viscosity value of $10.9 \mathrm{dL} / \mathrm{g}$ resulted from a sample prepared by stirring at room 
temperature overnight. The affect of the temperature of solvent preparation on the viscosity of the solution is likely due to the presence of the very high molecular weight, high acrylonitrile content, polymer chains being very slow to dissolve at room temperature, or the potential for aggregation of the polymer chains during the dissolution process. Table 2 provides the comparison of dilute solution viscosity measurements.

Based on the recognition that the addition of salt affected the polymer aggregation and molecular weight analysis by light scattering, the intrinsic viscosity in DMF with $0.05 \mathrm{M} \mathrm{LiBr}$ was measured to compare to the value obtained in DMF without salt. The intrinsic viscosity analysis did not show a large difference due to the addition of salt, exhibiting a value of $9.8 \mathrm{dL} / \mathrm{g}$ without salt and $9.7 \mathrm{dL} / \mathrm{g}$ with salt. Addition of the salt is proposed to break up aggregation of the polymer chains, which could affect polymer size and viscosity. The aggregation observed in the light scattering measurements (presented in Figure 5) was not detected in the intrinsic viscosity results.

Table 2. Dilute solution viscosity measurements of a sample of very high molecular weight polyacrylonitrile-co-methyl acrylate

\begin{tabular}{|c|c|c|c|}
\hline Sample & $\begin{array}{c}\eta_{\text {inh }}\left(\mathrm{DMF} \text { at } 35^{\circ} \mathrm{C}\right)(\mathrm{dL} / \mathrm{g}) \\
(0.1 \mathrm{~g} / \mathrm{dL} \text { concentration })\end{array}$ & $\begin{array}{l}{[\eta]^{35} \mathrm{DMF}} \\
(\mathrm{dL} / \mathrm{g})\end{array}$ & $\begin{array}{l}{[\eta]^{35} \text { DMF w/ 0.05 M }} \\
\text { LiBr (dL/g) }\end{array}$ \\
\hline 97/3 VHMW AN/MA & 10.9 & 9.8 & 9.7 \\
\hline
\end{tabular}

The samples of interest were VHMW copolymers with very high acrylonitrile content. Due to the high acrylonitrile content and high molar mass, the high viscosity of the solutions was apparent even at low concentrations. For the SEC measurements it was necessary to prepare the samples at relatively low concentrations of 0.5 to $1.5 \mathrm{mg} / \mathrm{mL}$ and use a larger than normal 1.6 $\mu \mathrm{m}$ filter for the sample solutions. Initially samples were run in NMP without added salt. Figure 5 presents the refractive index, light scattering, and viscosity chromatograms of a very high molecular weight sample run in NMP at $50{ }^{\circ} \mathrm{C}$. This sample was a 97/3 AN/MA copolymer (by weight) prepared by emulsion polymerization. The sample was prepared at about $0.5 \mathrm{mg} / \mathrm{mL}$ and filtered with a $1.6 \mu \mathrm{m}$ syringe filter. Light scattering was used to analyze the size of the molecules and the molar mass of the molecules. A dn/dc of 0.066 was used for the analysis. The $\mathrm{dn} / \mathrm{dc}$ was calculated from an experiment where the $\mathrm{dn} / \mathrm{dc}$ was determined offline in batch mode for a series of four AN/MA copolymers with varying molecular weights and AN content from 85 to $97 \%$ in NMP/0.05 M LiBr.

Figure 5 shows that the concentration and viscosity profiles, as shown by the refractive index and viscometer chromatographic results, differ from the light scattering profile with elution time. The light scattering peak appears to be bimodal with a very intense peak eluting first and a smaller peak that coincides with the bulk of the concentration of the sample eluting later. The very intense peak has no appreciable concentration or viscosity to it, indicating that it is a result of the presence of a relatively low concentration of very large/high molar mass species. In fact, the species in the early eluting intense light scattering peak are estimated to be on the order of $200 \mathrm{~nm}$ in size by the MALLS system. The same sample was run in NMP with $0.05 \mathrm{M} \mathrm{LiBr}$ added in order to eliminate or reduce polar interactions causing polymer aggregation and column exclusion (17). 


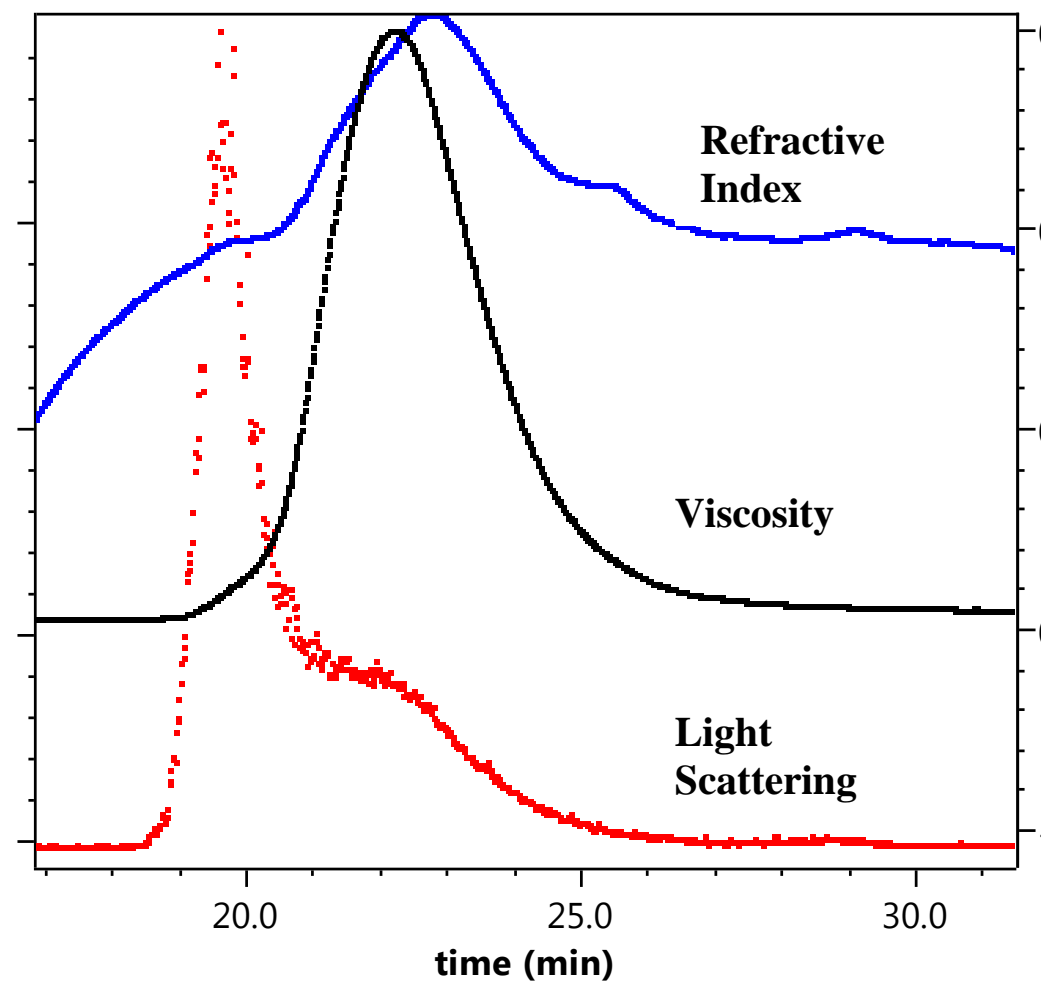

Figure 5. SEC chromatograms of a 97/3 AN/MA copolymer in NMP at $50^{\circ} \mathrm{C}$ where the sample was filtered through a $1.6 \mu \mathrm{m}$ filter (SEC analysis results are provided in Table 3 )

Table 3 provides a comparison of the molecular weight analysis for various solvent, concentration, and column configurations for the same sample of polymer powder. The molecular weight analysis provided in Table 3 consists of the molar mass averages, polydispersity, and average radius of each sample run by SEC for comparison. The injected mass is related to the concentration of a 100-microliter injection, and the mass recovery is related to the weight percent of the sample that was detected in the eluent by the refractive index detector. Loss of material is typically due either to filtration of undissolved or very large aggregated material during the sample preparation, or it can be due to polymer chains interacting with and sticking on the columns. Therefore, sample concentration, solvent composition, and column type were all found to be critical factors to the SEC measurements. The addition of the salt clearly results in a significant decrease in polymer aggregation in solution but the low mass recovery values indicate that material is still being lost during these SEC runs which may be limiting the range of the molecular weight distribution that is included in the molecular weight analysis. Higher salt compositions were prepared to further reduce the aggregation of the polymer in solution but these tests were not successful due to the limited solubility of the lithium salt in NMP.

Figure 6 shows the comparison of the light scattering chromatograms of the samples run through the same set of columns with and without $\mathrm{LiBr}$ added to the NMP. The results of the sample in NMP without added salt indicate that aggregation of the polymer molecules is occurring in this solvent system and that it can be at least reduced by the addition of $\mathrm{LiBr}$ salt. The results show low mass recoveries, which varies somewhat from sample to sample. The same sample preparation procedures used concentrations ranging from 0.5 to $1.5 \mathrm{mg} / \mathrm{mL}$. It is seen that the 
average molar mass values, as calculated by light scattering, decrease with decreasing mass recovery and there is an inverse correlation between sample concentration and mass recovery. This indicates that the high molecular weight fractions are being removed from the sample either by filtration of insoluble or large molecules during the sample preparation or by filtration in the columns. Therefore, it appears that the true molecular weight of this polymer is elusive because only the average molar mass of the smaller unfiltered fraction of the molecules can be measured due to the restrictions of the sample preparation and method conditions. Even with this limitation, it was possible to determine that the weight average molar mass of the polymer is at least 1.7 million $\mathrm{g} / \mathrm{mole}$.

Table 3. SEC analysis of the same 97/3 AN/MA polymer sample in NMP with and without 0.05 $\mathrm{M} \mathrm{LiBr}$ at $50{ }^{\circ} \mathrm{C}$ using two different columns shows the impact of losing the high molecular weight fraction of sample on the molecular weight results.

\begin{tabular}{|c|c|c|c|c|c|c|c|}
\hline $\begin{array}{c}\text { Solvent/ } \\
\text { Column }\end{array}$ & $\begin{array}{c}\mathrm{M}_{\mathrm{n}} \\
(\mathrm{kDa})\end{array}$ & $\begin{array}{c}\mathrm{M}_{\mathrm{w}} \\
(\mathrm{kDa})\end{array}$ & $\begin{array}{c}\text { Polydispersity } \\
\left(\mathrm{M}_{\mathrm{w}} / \mathrm{M}_{\mathrm{n}}\right)\end{array}$ & $\begin{array}{c}\mathrm{Rz} \\
(\mathrm{nm})\end{array}$ & $\begin{array}{c}\text { Injected } \\
\text { mass }(\mu \mathrm{g})\end{array}$ & $\begin{array}{c}\text { Mass } \\
\text { recovery }(\%)\end{array}$ & $\begin{array}{c}\mathrm{dn} / \mathrm{dc} \\
(\mathrm{mL} / \mathrm{g})\end{array}$ \\
\hline $\begin{array}{c}\text { NMP/1 } \\
\text { high MW } \\
\text { mixed bed } \\
\text { PMA }\end{array}$ & 2193 & 13,844 & 6.31 & 218 & 56 & 73 & 0.066 \\
\hline $\begin{array}{c}\text { NMP0.05M } \\
\text { LiBr/3 } \\
\text { Mixed Bed } \\
\text { PS }\end{array}$ & 952 & 1514 & 1.59 & 113 & 86 & 52 & 0.066 \\
\hline $\begin{array}{c}\text { NMP0.05M } \\
\text { LiBr/2 high } \\
\text { MW mixed } \\
\text { bed PMA }\end{array}$ & 634 & 1268 & 2.00 & 131 & 148 & 34 & 0.066 \\
\hline $\begin{array}{c}\text { NMP0.05M } \\
\text { LiBr/2 high } \\
\text { MW mixed } \\
\text { bed PMA }\end{array}$ & 1064 & 1713 & 1.61 & 111.7 & 67 & 52 & 0.066 \\
\hline
\end{tabular}

The polymers of interest for this effort have significantly higher molar masses than commercially spun polymers used to produce carbon fiber precursors. SEC was used to compare a commercial copolymer, obtained by Oak Ridge National Laboratories, to the VHMW sample. The normalized light scattering chromatograms and weight average molar mass values are provided in Figure 7. The very high molecular weight polymer elutes earlier and the relative light scattering response is observed to be significantly larger due to the larger size of the molecules in solution.

Due to the fact that the samples appear to aggregate in NMP, it seems likely that the same behavior is present in related solvents such as DMF, DMAc, and DMSO, which are often used for solution spinning these polymers. The spinning solutions of these polymers in DMAc were prepared at a concentration of around $6 \mathrm{wt} \%$ solids and initially filtered through a $140 \mu \mathrm{m}$ filter which is likely large enough to allow aggregates to pass through. It was observed that the material had a very high viscosity and the gelled fibers were mechanically very coherent, leading to high drawability. The presence of a highly aggregated/entangled component, as seen in the 
SEC in NMP without LiBr, could be adding to the strength of the gel phase. Molecular weight analysis of unfiltered very high molecular weight polyacrylonitrile copolymers is a subject of research in the laboratories at Virginia Tech utilizing Asymmetric Flow Field Flow Fractionation (AF4) equipment in NMP provided through an NSF-MRI.

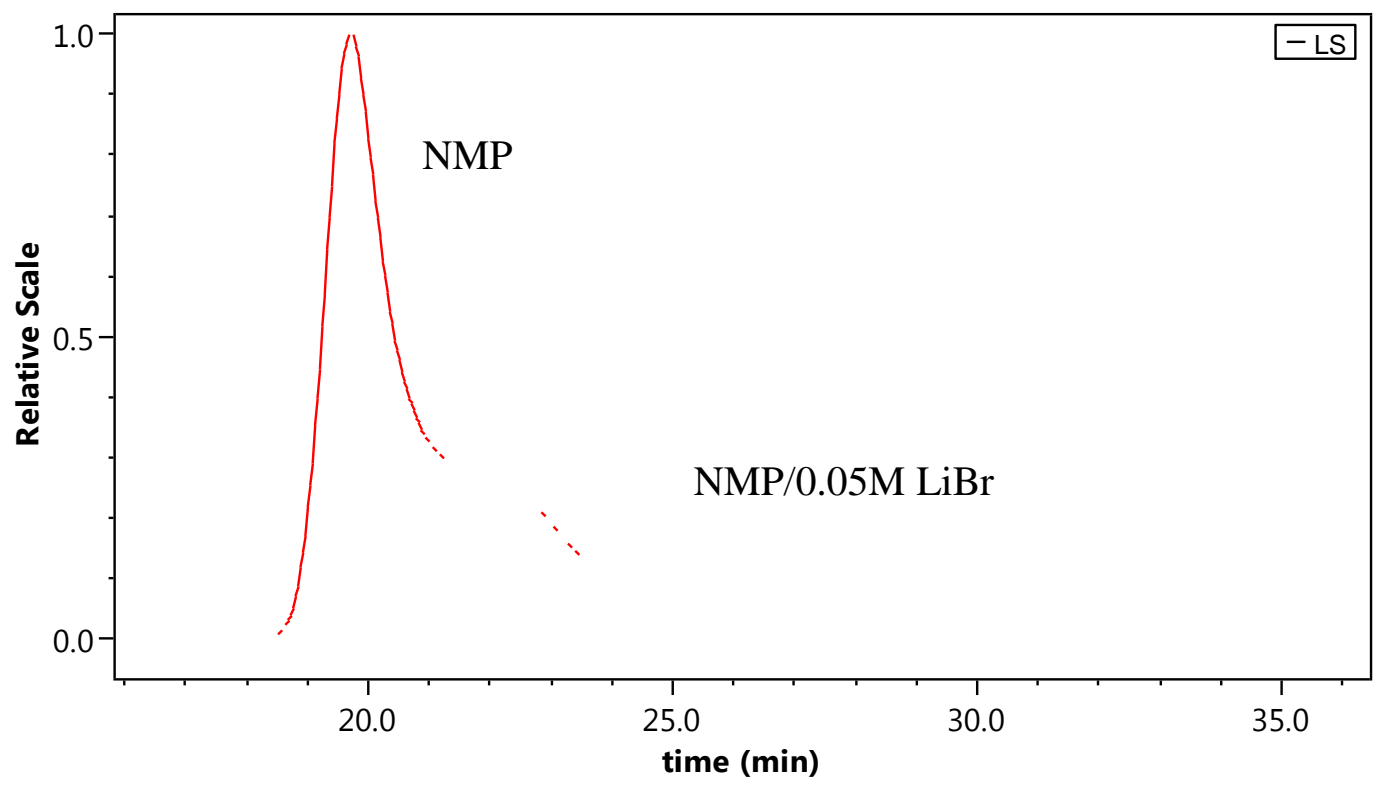

Figure 6. Aggregation is apparent in the comparison of the normalized MALLS chromatograms of 97/3 AN/MA copolymer run in NMP with and without $\mathrm{LiBr}$ salt added.

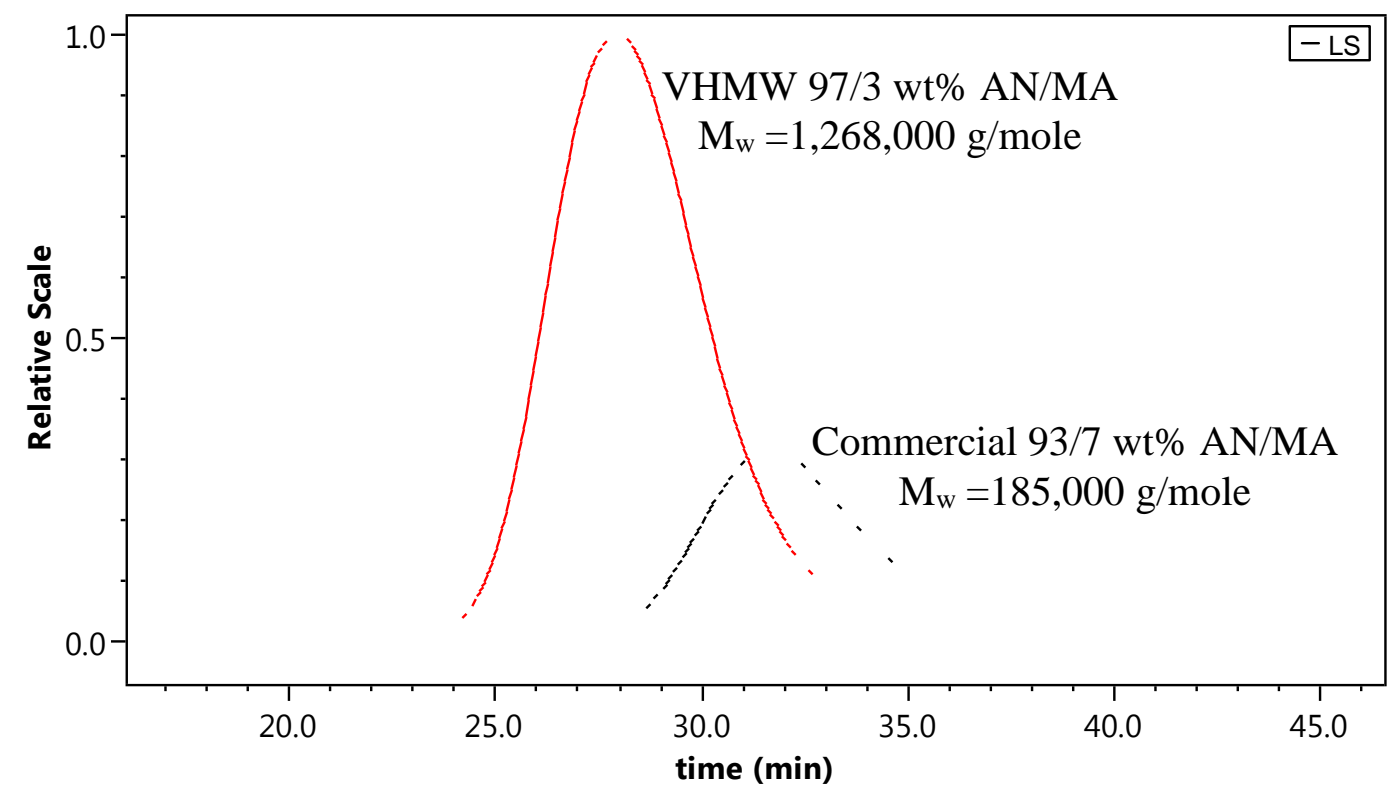

Figure 7. Normalized light scattering chromatograms of a Virginia Tech synthesized VHMW 97/3 AN/MA compared to a commercially prepared 93/7 AN/MA copolymer run in NMP with $0.05 \mathrm{M} \mathrm{LiBr}$. 


\subsection{Viscoelastic Properties of the VHMW PAN-co-MA Dope}

Virginia Tech-synthesized VHMW (approximately 1.7 million $\mathrm{M}_{\mathrm{w}}$ ) 97\% acrylonitrile, 3\% methyl acrylate (by weight) copolymer was provided to UKY CAER for spinning trials. Initial formulation of the polymer-to-solvent concentration for spinning dopes relied on a previously established internal database of experimental data of spinnable solution viscosities. Steady shear viscosities of 150-200 Pa-sec at $1 \mathrm{~s}^{-1}$ shear rate and $25{ }^{\circ} \mathrm{C}$ were targeted for dry-jet (or air-gap) spinning. As formulation of VHMW polymer dopes continued, a more accurate determination of spinnable viscoelastic property ranges was established for these VHMW polymers by rheological methods.

The results for several spinnable VHMW polymer dopes can be found in Figure 8. The results show shear thinning occurring immediately at very low shear rates, similar to the results found by Jiang (18). The results also provide a wide target-window for steady state shear viscosity, ranging from 70 to $250 \mathrm{~Pa}-\mathrm{sec}$ at $1 \mathrm{~s}^{-1}$ shear rate at $25^{\circ} \mathrm{C}$.

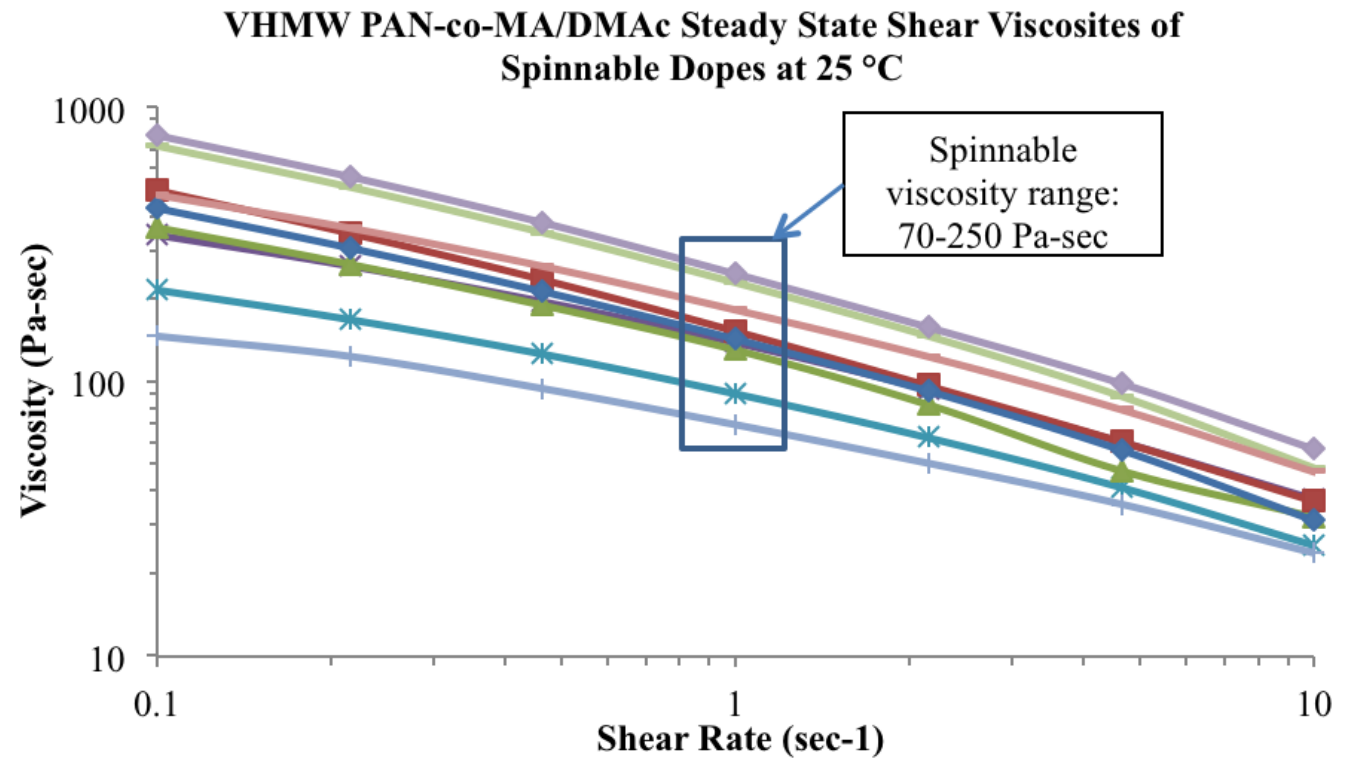

Figure 8. Steady state shear viscosities of VHMW polymer dopes as a function of shear rate at $25^{\circ} \mathrm{C}$

As dope formulation trials continued, the parameter ranges acceptable for good spinnability for other rheological properties of the dopes emerged, which included the storage and loss shear moduli, $G^{\prime}$ and $G^{\prime \prime}$ respectively, as a function of temperature, shown in Figure 9. Storage and loss shear moduli decrease with an increase in temperature, as expected based on the results of Jiang (18). In addition, a crossover point of the storage and loss shear moduli was typically observed for these VHMW PAN-based dopes. At room temperature, the dope behaved as a gel, as the elastic solid-like behavior quantified by the storage shear modulus, $G^{\prime}$, was larger than the viscous liquid-like behavior quantified by the loss shear modulus, $G^{\prime \prime}$. However, as the temperature was increased, the dope became increasingly viscous as opposed to elastic, with a crossover occurring at $72{ }^{\circ} \mathrm{C}$ and $130 \mathrm{~Pa}$. Similarly, as the temperature decreased below ambient conditions, the dope became increasingly elastic, with the $G^{\prime}$ further diverging from $G^{\prime \prime}$. 


\section{6 wt \% VHMW PAN-co-MA Dope Modulus vs. Temperature}

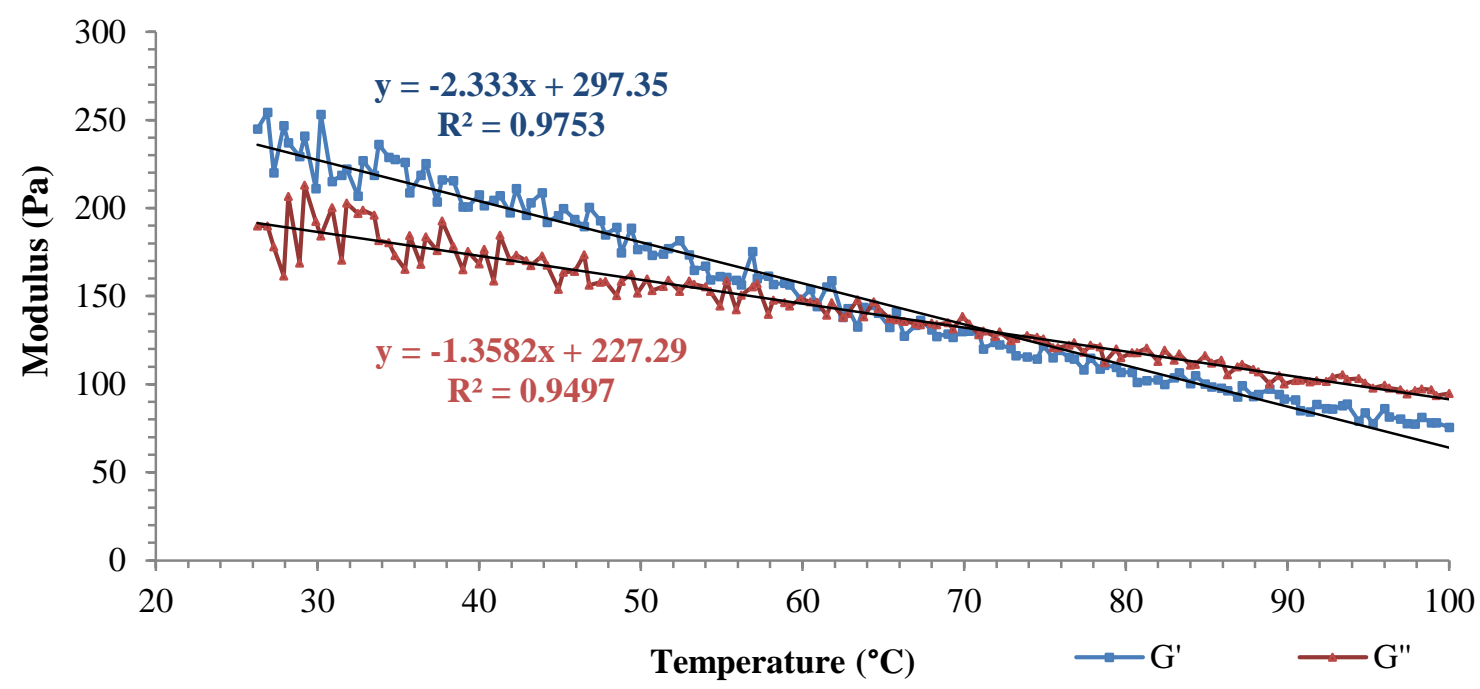

Figure 9. The storage and loss shear moduli as a function of temperature with an angular frequency of $10 \mathrm{rad} / \mathrm{s}$ for dope showing the crossover point of $G^{\prime}$ and $G^{\prime \prime}$

All of the results from these tests were compiled and analyzed, resulting in the summary graph shown in Figure 10, which details the average spinnable windows for various rheological properties of the spinning dopes. The average steady shear viscosity at $1 \mathrm{~s}^{-1}$ and $25{ }^{\circ} \mathrm{C}$ ranged from 250 to $70 \mathrm{~Pa}-\mathrm{s}$. The average $G^{\prime}$ at $10 \mathrm{rad} / \mathrm{s}$ and $25^{\circ} \mathrm{C}$ was between 380 and $142 \mathrm{~Pa}$, and similarly the average $G^{\prime \prime}$ was between 280 and $125 \mathrm{~Pa}$. Average moduli were calculated using the linear regression equation from the temperature sweep data, similar to Figure 9. The average crossover temperature where $G^{\prime}$ equaled $G^{\prime \prime}$ in magnitude was between 118 and $64{ }^{\circ} \mathrm{C}$. The modulus at this crossover point was determined to lie between 138 and $86 \mathrm{~Pa}$. All of these spinnable ranges were important to formulating and developing spinnable dopes.

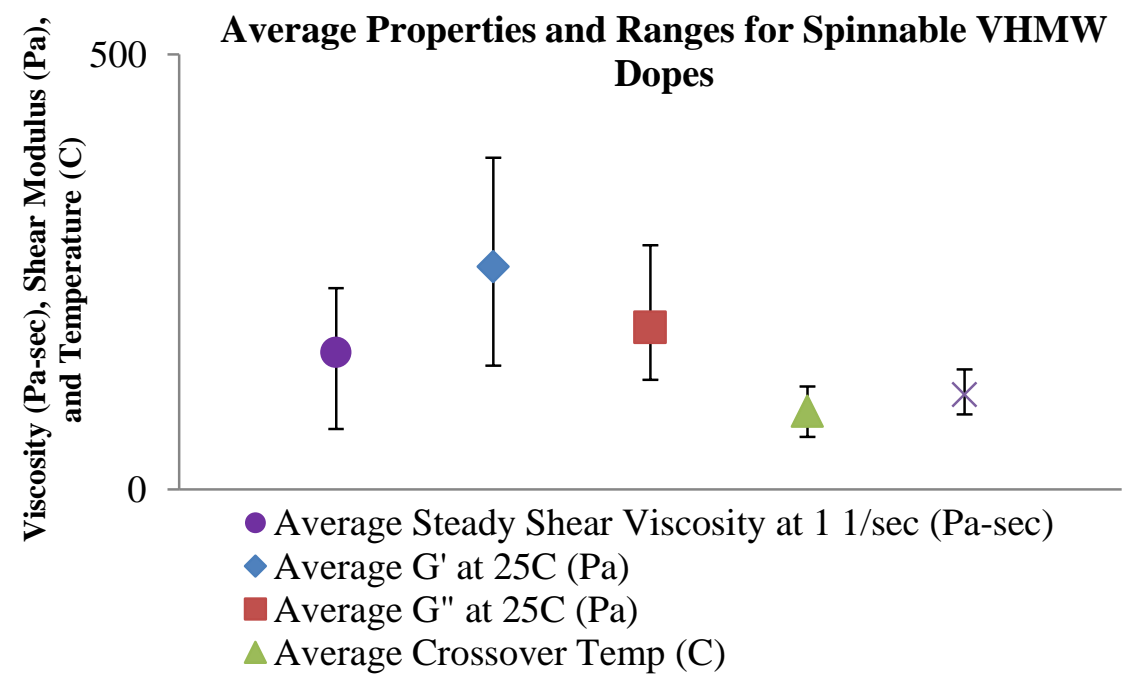

Figure 10. Spinnable windows for rheological properties determined experimentally 
Further rheological analyses on formulated dopes was necessary to diagnose and correct issues related to spinnability. Initial spinning trials with VHMW polymer dope resulted in difficult startup for dry-jet spinning. A $6 \mathrm{wt} \%$ solution of VHMW PAN-co-MA in DMAc was spun into a $60 \mathrm{wt} \% \mathrm{DMAc} / \mathrm{H}_{2} \mathrm{O}$ coagulation bath maintained at $8^{\circ} \mathrm{C}$. Nascent filaments did not properly coagulate and began to fail cohesively under small amounts of process tension in subsequent wash baths. Initial efforts focused on increasing the coagulation bath temperature to speed up coagulation kinetics. Solvent and water diffusion rates out of and into the polymer, respectively, have been shown to increase with bath temperature (2). However, repeated attempts with the coagulation bath temperature increased to $30^{\circ} \mathrm{C}$ resulted in similar failures.

Oscillatory rheology results indicated a relatively high storage (elastic) shear modulus $\left(G^{\prime}\right)$, which contributed to the solid-like, or gel behavior (Figure 11), particularly at lower temperatures. A spinnable lower molecular weight dope (LMW) $\left(\mathrm{M}_{\mathrm{w}} \approx 200,000\right)$ at $20 \mathrm{wt} \%$ concentration showed the loss shear modulus $\left(G^{\prime \prime}\right)$ well above the storage shear modulus in magnitude, suggesting that the liquid-like behavior of the solution dominated, allowing diffusion to take place more readily (Figure 12) (19). Due to the divergent nature of the storage and loss moduli for VHMW PAN-co-MA with decreasing temperature, it was hypothesized that spinning into a chilled coagulation bath should drive the spinning solution towards a more gel-like state, and therefore create a more tenacious nascent filament - better able to withstand the process tensions encountered during the early coagulation and washing processes, during which solvent migration could also occur. Therefore, it was discovered that spinning into a coagulation bath near $0{ }^{\circ} \mathrm{C}$ was successful for the VHMW polymer solutions. Spinning polymer solutions into chilled baths to cause a temperature driven phase inversion is often referred to as gel spinning $(20,21)$. Previous polyacrylonitrile gel spinning attempts have produced high strength fibers $(1.8 \mathrm{GPa})$, but with large diameters $(100 \mu \mathrm{m})$. The large diameter hindered thermal treatment and required excessive processing time, as fibers had to remain submerged in a bath for two days to fully solidify, limiting production capabilities (22). The proposed hybrid method in this work between dry-jet and gel spinning will allow production of small diameter, high strength fibers using VHMW PAN-co-MA polymers and processing techniques suited for continuous operation.

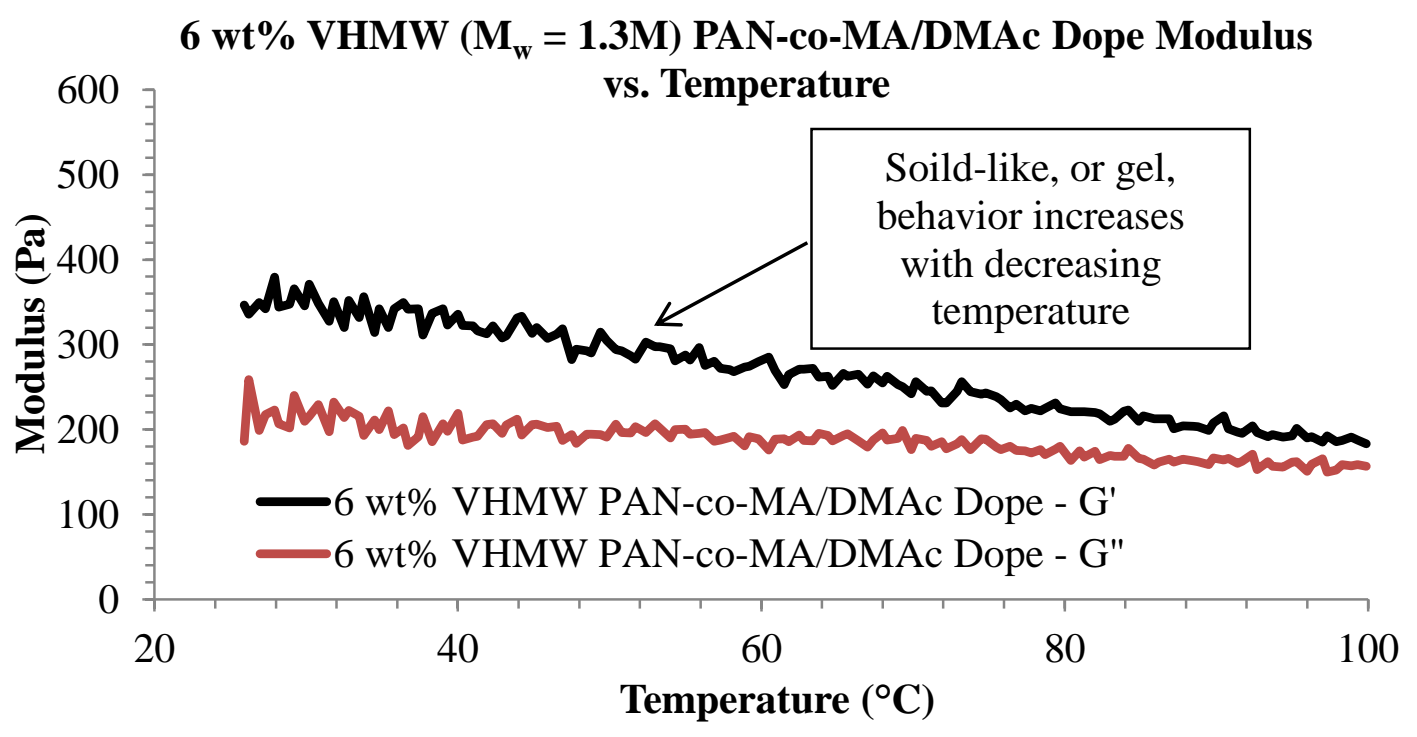

Figure 11. Oscillatory rheology of a VHMW polymer solution $\left(\mathrm{M}_{\mathrm{w}}=1.3 \mathrm{M}\right)$ 


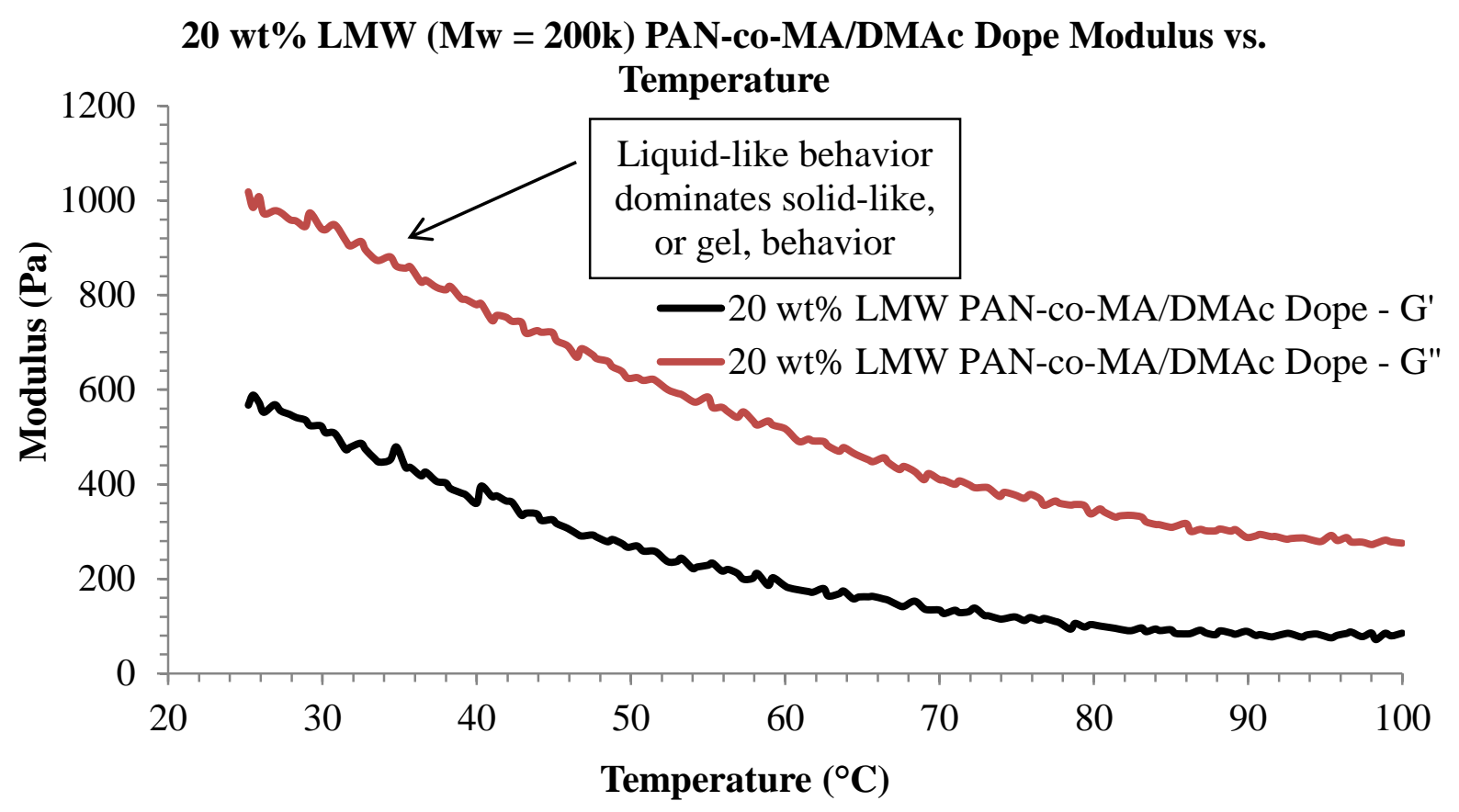

Figure 12. Oscillatory rheology of a LMW PAN-co-MA polymer solution $\left(\mathrm{M}_{\mathrm{w}}=200 \mathrm{k}\right)$ showing marked differences in the storage and loss shear moduli as a function of temperature compared to the VHMW PAN (Figure 11).

\subsection{VHMW PAN-co-MA Fiber Spinning Conditions}

As described previously, a variation of the dry-jet (or air-gap) solution spinning method was used to produce filaments from VHMW polymers, termed "hybrid dry-jet gel spinning". Low coagulation bath temperatures were used to drive the forming filaments toward a gel state resulting in more tenacious nascent filaments.

The drawdown ratio (DDR) that the fiber tow was subjected to was measured between each set of godet drives (while within the preceding bath). The highest amount of stretching (DDR = 5.75) occurred in the coagulation bath. However, much of this draw occurred in the air gap. The dry-jet spinning method enabled high spin draw of the emerging polymer jets. The gel-like nature of the emerging filaments at this stage in the process also enabled high amounts of stretch, as the PAN molecules retained high mobility in the solution and were not yet set in place by the coagulation process. The forming, gelled filaments were further capable of withstanding a 1.50 DDR in the first wash bath and 1.33 DDR in the second wash bath, after which the fiber was solidified to the point that stretching within the subsequent cool and room temperature wash baths was not possible without producing fiber breakage. After washing, stretching occurred in a hot water bath, and subsequently in a heated glycerol bath, with DDRs of 1.70 and 2.20, respectively. The remaining portion of the line was not used for stretching, but rather for preparing the fiber for take-up. Measurements of residual DMAc showed a gradual, linear decrease in DMAc concentration in the filaments as they progressed through the coagulation/wash baths, resulting in a statistically zero residual content, which confirmed that the number of baths and selection of concentrations was appropriate. 
Table 4 provides the spin draw (the stretch the fiber encountered in the air gap and coagulation bath), as well as the fiber stretch experienced throughout the remaining portion of the line (this is calculated as the product of the subsequent DDRs). The total DDR is the product of the spin draw and the fiber stretch, and for a representative VHMW PAN-co-MA, was determined to be 43.0. This high drawdown ratio (16), coupled with low solids content in the dope, led to precursor filament diameters of approximately $5 \mu \mathrm{m}$ (equivalent circular diameter). An example of the collected precursor fiber in Figure 13 shows a 100 filament count tow, $560 \mathrm{~m}$ long, with a $5.7 \pm 0.4 \mu \mathrm{m}$ filament diameter, $1037 \pm 101 \mathrm{MPa}$ tensile strength, and $17.9 \pm 1 \mathrm{GPa}$ tensile modulus.

Table 4. The product of spin draw and fiber stretch give the total drawdown ratio (DDR)

\begin{tabular}{|c|c|c|}
\hline Spin Draw & Fiber Stretch & TOTAL DDR \\
\hline 5.75 & 7.5 & $\mathbf{4 3 . 0}$ \\
\hline
\end{tabular}

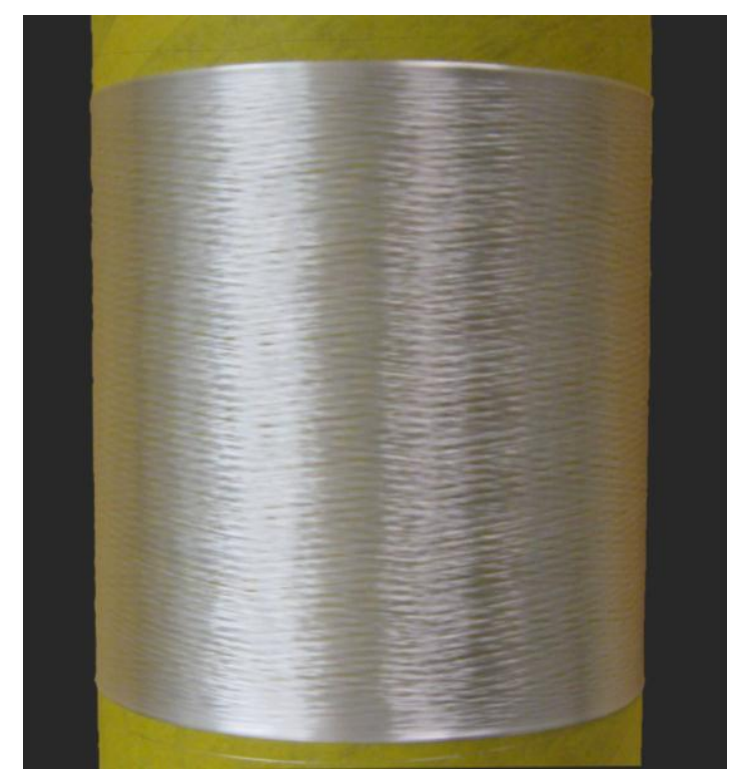

Figure $13.560 \mathrm{~m}$ of hybrid dry-jet, gel spun VHMW PAN-co-MA fiber in 100 filament count tow $(5.7 \pm 0.4 \mu \mathrm{m}$ filament diameter, $1037 \pm 101 \mathrm{MPa}$ tensile strength, and $17.9 \pm 1 \mathrm{GPa}$ elastic modulus)

\subsection{As-Spun Fiber Structure and Physical Properties}

As shown in Figure 14, the as-spun filaments had a slight kidney bean shape. Bean shapes can be common among solution spun PAN fibers, particularly when spun from dopes with low polymer solids and high $\mathrm{M}_{\mathrm{w}}$ polymers $(3,11,23)$. In addition, utilizing a cold coagulation bath, although necessary for this process, favors solvent flux out over water flux into the nascent fibers, also contributing to bean shapes $(15,24,25,26)$. 


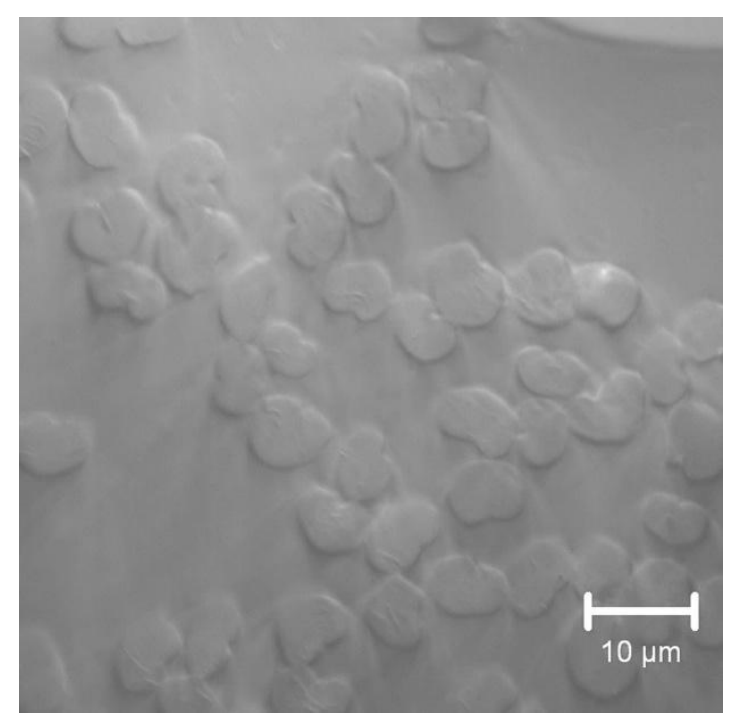

Figure 14. Reflected light optical microscopy of VHMW PAN-co-MA fiber ends embedded in epoxy, magnification 1000x

The fiber cross section shown in Figure 15 clearly shows the bean shape of the fibers, as well as some skin formation on the fiber surface, indicated by the arrows. This skin formation is likely a result of moisture-induced phase separation in the air gap $(27,28,29,30)$.

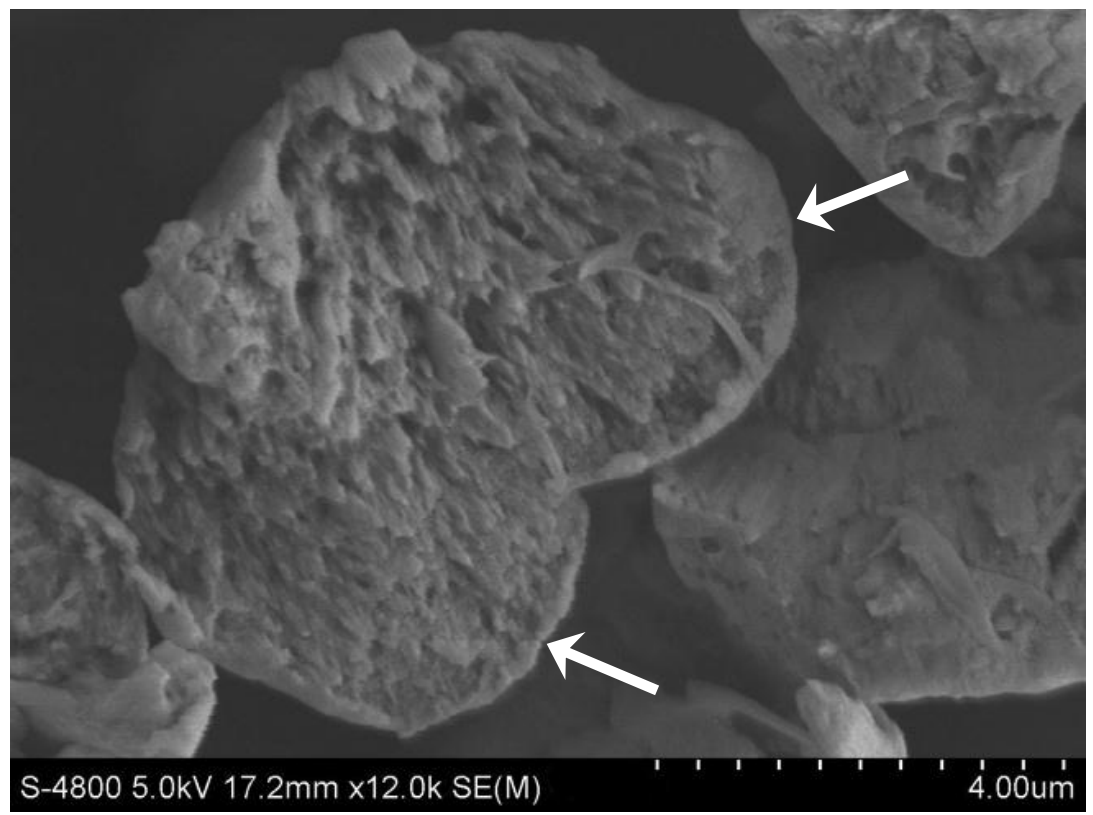

Figure 15. Precursor fiber cross section imaged using SEM

The images in Figure 16 show the smooth, near defect-free surfaces of fibers spun using the dryjet gel spinning hybrid technique. It also highlights their bean-shape. In Figure 16a and b, the bean-shape is indicated by the arrows, which point to the concavity along the fiber midsection. Figure 16c shows the smooth fiber surface, typical of dry-jet spun fibers (31). Figure 16d is a high magnification $(100 \mathrm{kx})$ of the fiber surface, showing striations along the fiber surface parallel to the fiber axis. This is characteristic of solution spun fibers which undergo diffusion 
during the coagulation process and fibril orientation on the fiber surface $(32,33)$. Overall, the filament surfaces were smooth and relatively defect-free, which contributed to their high tensile properties.

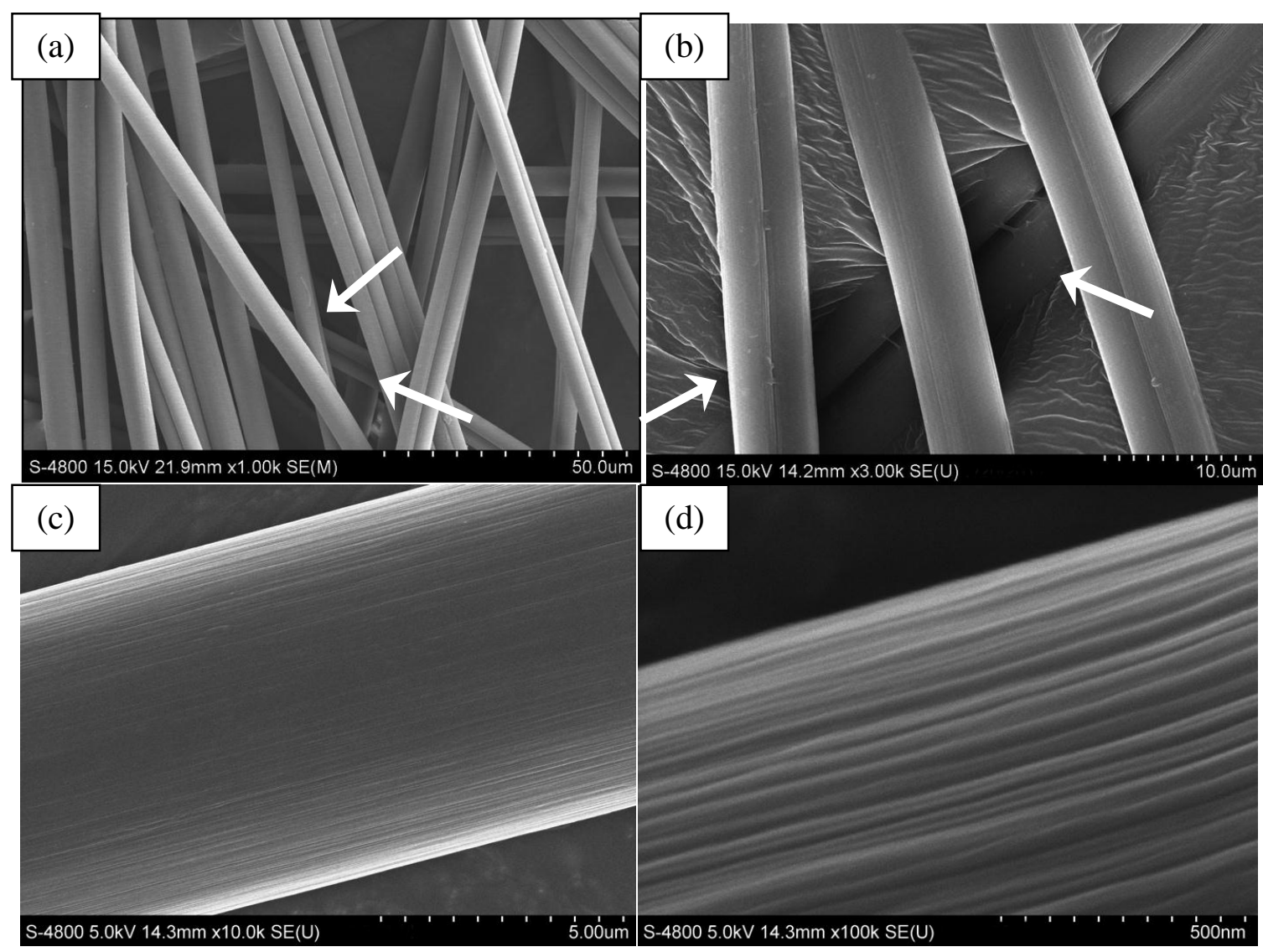

Figure 16. SEM images of dry-jet spun VHMW PAN-co-MA fiber. (a) Fibers at 1000x magnification (bean-shape highlighted by arrows). (b) Fibers at 3000x magnification (beanshape highlighted by arrows). (c) 10kx magnification shows smooth fiber surface, typical of dryjet spun fibers. (d) High magnification $(100 \mathrm{kx})$ shows striations in the fiber surface, typical for fibers produced using a diffusion coagulation process

Tensile properties of commercial precursor filaments are not widely available. However, details about tensile strength, elastic modulus, and diameter can be gleaned from literature. For example, Hexcel produced a high performance carbon fiber with a tensile strength of $6.7 \mathrm{GPa}$ and elastic modulus of $324 \mathrm{GPa}$ from a relatively small precursor fiber diameter of 0.6 to 0.8 den, or 8.5 to $9.8 \mu \mathrm{m}$ (34), assuming a filament density of $1.18 \mathrm{~g} / \mathrm{cc}$. Japan Exlan Company produced a PAN fiber having a larger diameter of 50 to $300 \mu \mathrm{m}$ and a lower tensile strength of 80 to $460 \mathrm{MPa}$, from a polymer with a $\mathrm{M}_{\mathrm{w}} \geq 400,000$ (35). Toray Industries produced acrylic precursor fibers with $7.1 \mathrm{~g} / \mathrm{den}$ strength. They report a resulting carbon fiber with a single filament diameter of $7.0 \mu \mathrm{m}$. Assuming a $50 \%$ reduction in fiber diameter from acrylic to carbon $(36,37,38,39,40)$, it can be estimated that the precursor fibers had a diameter of approximately $14 \mu \mathrm{m}$. Based on this diameter, a $7.1 \mathrm{~g} /$ den strength translates to a precursor tensile strength of $456 \mathrm{MPa}$ (41). Mitsubishi Rayon precursor fibers were reported to have a tensile strength of 579 
MPa (42). Based on a titre of 1.24 dtex, the diameters of those fibers would have been $12.9 \mu \mathrm{m}$ (42). A patent by Nikkiso Co., Ltd. described production of a precursor filament with a diameter of $6.3 \mu \mathrm{m}$ and a tensile strength of $687 \mathrm{MPa}$ by spinning a $5.5 \mathrm{wt} \%$ solution of PAN with $\mathrm{M}_{\mathrm{w}}=$ 130,000 (43). Based on these commercial examples, typical precursor fibers are generally no less than $6.3 \mu \mathrm{m}$, with a tensile strength no greater than $687 \mathrm{MPa}$, as summarized in Table 5 .

Table 5. Summary of precursor fiber diameters and tensile strengths produced with reference to commercial manufacturer

\begin{tabular}{c|c|c|c}
\hline Manufacturer & $\begin{array}{c}\text { Precursor Fiber } \\
\text { Diameter }(\mu \mathrm{m})\end{array}$ & $\begin{array}{c}\text { Precursor Fiber Tensile } \\
\text { Strength (MPa) }\end{array}$ & Reference \\
\hline \hline Japan Exlan & $50-300$ & 80 to 460 & $(35)$ \\
Hexcel & $8.5-9.8$ & 456 & $(34)$ \\
Toray Industries & 14 & 579 & $(41)$ \\
Mitsubishi Rayon & 12.9 & 687 & $(42)$ \\
Nikkiso Co, Ltd. & 6.3 & & $(43)$
\end{tabular}

In this work, filament diameter was shown to have a major influence on the tensile properties of the filaments (Figure 17). Smaller diameter filaments had higher tensile properties. This is in accordance with Griffith's fracture theory, which is consistent with the statement that at its extreme, a single polymer chain (filament of minimum diameter) would represent the strongest, highest-modulus filamentous form because such a filament could not exist with any defects present (44), and would contain no fraction of misoriented chains.

Figure 17 shows a power law relationship between ultimate break stress and fiber diameter, with $\mathrm{R}^{2}=0.91$. The tensile strength and elastic modulus of the smaller diameter filaments, measuring from 4 to $6 \mu \mathrm{m}$, were averaged in Figure 18 and found to be $954 \pm 103 \mathrm{MPa}$ in strength, $15.9 \pm$ $1.8 \mathrm{GPa}$ in elastic modulus $(\mathrm{N}=296)$ and $5.1 \pm 0.5 \mathrm{um}$ in diameter. Fundamentally, it is suggested that the use of VHMW polymers enabled the production of highly oriented, high tensile performance, small diameter filaments because: a) the very high molecular weight of the PANco-MA maintained the desired spinning viscosity even at low solids content. Less polymer per unit volume of nascent fiber results in commensurately smaller diameter filaments; b) the high molecular weight of the polymer chains was amenable to high degrees of draw which attenuated the fiber and increased the degree of chain orientation. Therefore, together, increased drawing of the fibers and lower solids content allowed for the production of a precursor filament diameter less than that used commercially, with a high tensile strength approaching $1 \mathrm{GPa}$ (compared to approximately $687 \mathrm{MPa}$ for commercial precursors). 
Filament Diameter Effect on Precursor Tensile Results

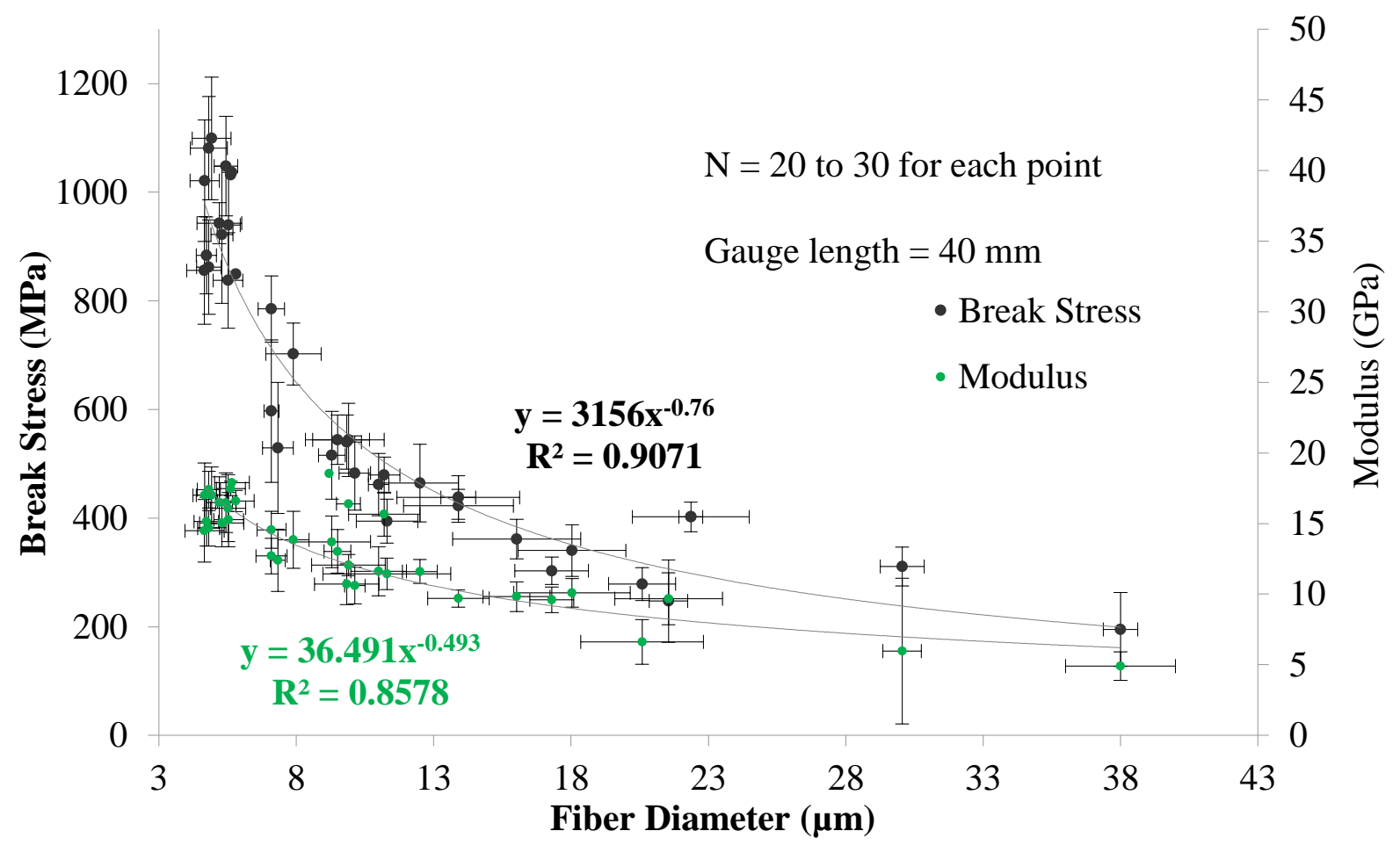

Figure 17. Tensile properties of precursor dry-jet, gel spun fibers in this work from VHMW PAN-co-MA

Fiber Tensile Properties for Filament Diameters in the 4-6 $\mu \mathrm{m}$ Range

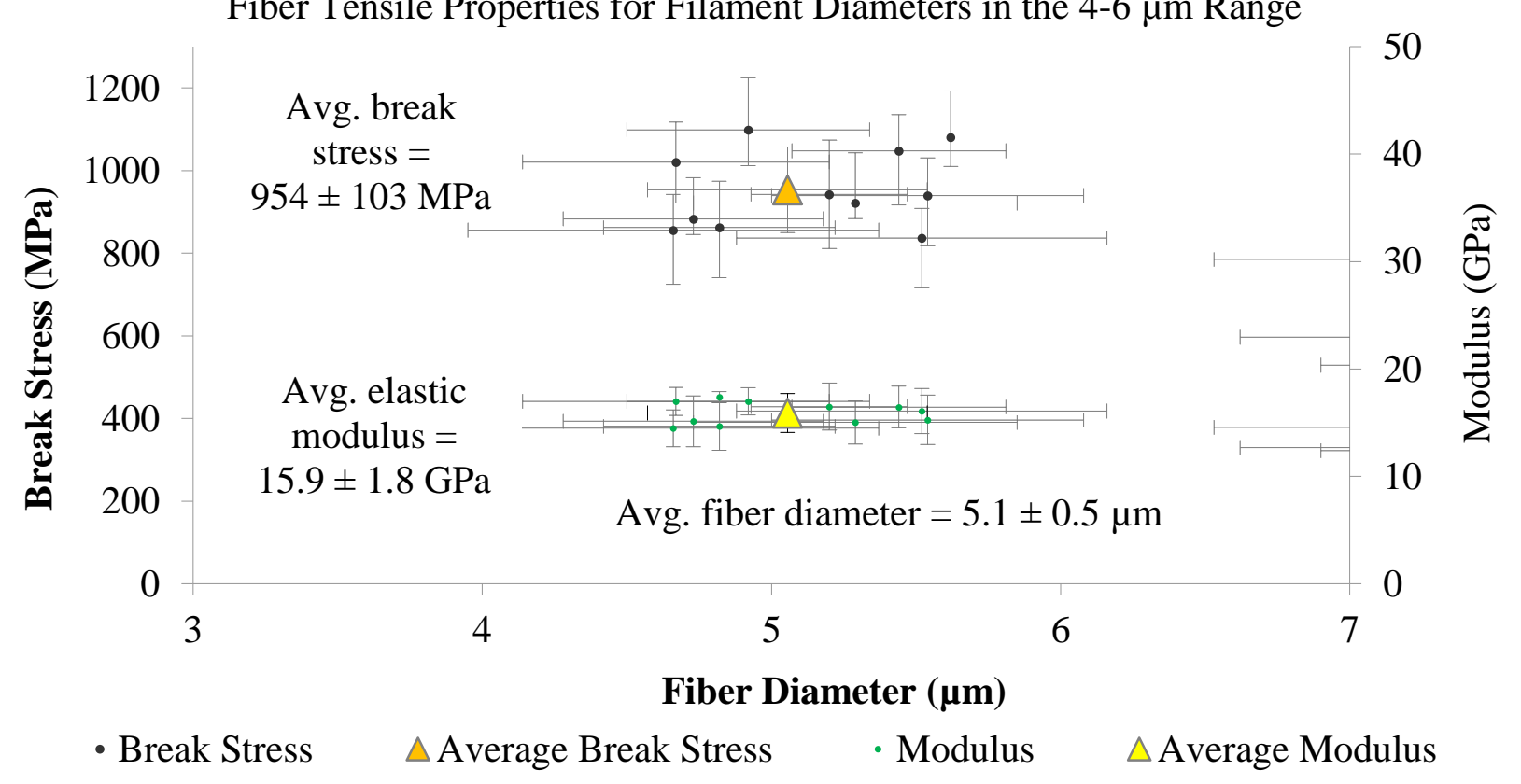

Figure 18. Fiber tensile properties for filament diameters in the 4-6 $\mu \mathrm{m}$ range, showing the average values for break stress and modulus 


\subsection{Effect of Polymer Molecular Weight on As-Spun Fiber Properties}

To illustrate the effect of molecular weight on fiber tensile properties, Figure 19 shows the results from Figure 17 separated based on polymer molecular weight, with high molecular weight characterized as being greater than 1 million Daltons $(1 \mathrm{M}) \mathrm{M}_{\mathrm{w}}$ and low molecular weight being less than $1 \mathrm{M} \mathrm{M}_{\mathrm{w}}$. Results indicated an overlap between both modulus and tensile strength for high and low molecular weight. One run consisted of an $18.5 \mathrm{wt} \%$ low molecular weight (LMW) polymer in DMAc with a high drawdown ratio of 40x, while another consisted of a 5 wt\% VHMW polymer in DMAc, intentionally drawn to a low drawdown ratio of only 5x (compared to a typical DDR = 43). It should be noted that although the VHMW polymer solution was drawn to a much lower drawdown ratio, it achieved the same fiber diameter as the lower molecular weight solution, which was drawn to a higher drawdown ratio. This is due to reduced solids content in the VHMW polymer dope. Therefore, while the runs consisted of differing molecular weights drawn at different ratios, the diameters, tensile strengths, and moduli overlapped, suggesting fiber tensile performance was not completely dependent on molecular weight.

In fact, the results indicated that fiber diameter played a larger role in determining final fiber tensile properties than molecular weight. However, as Figure 19 demonstrates, it is the high molecular weight polymer which enabled the production of the smallest filaments. That is, spinning dopes made with high molecular weight polymers could be prepared in a spinnable viscosity and processed at a lower solids content than lower molecular weight polymers. Also, high molecular weight polymers, due to the increased length of polymer chains, tended to have higher drawability (45), thus enhancing orientation along the length of the fiber. Low solids content in the spinning solution coupled with increased drawability led to smaller precursor fiber diameters, and as a result, precursor fibers with higher tensile performance.

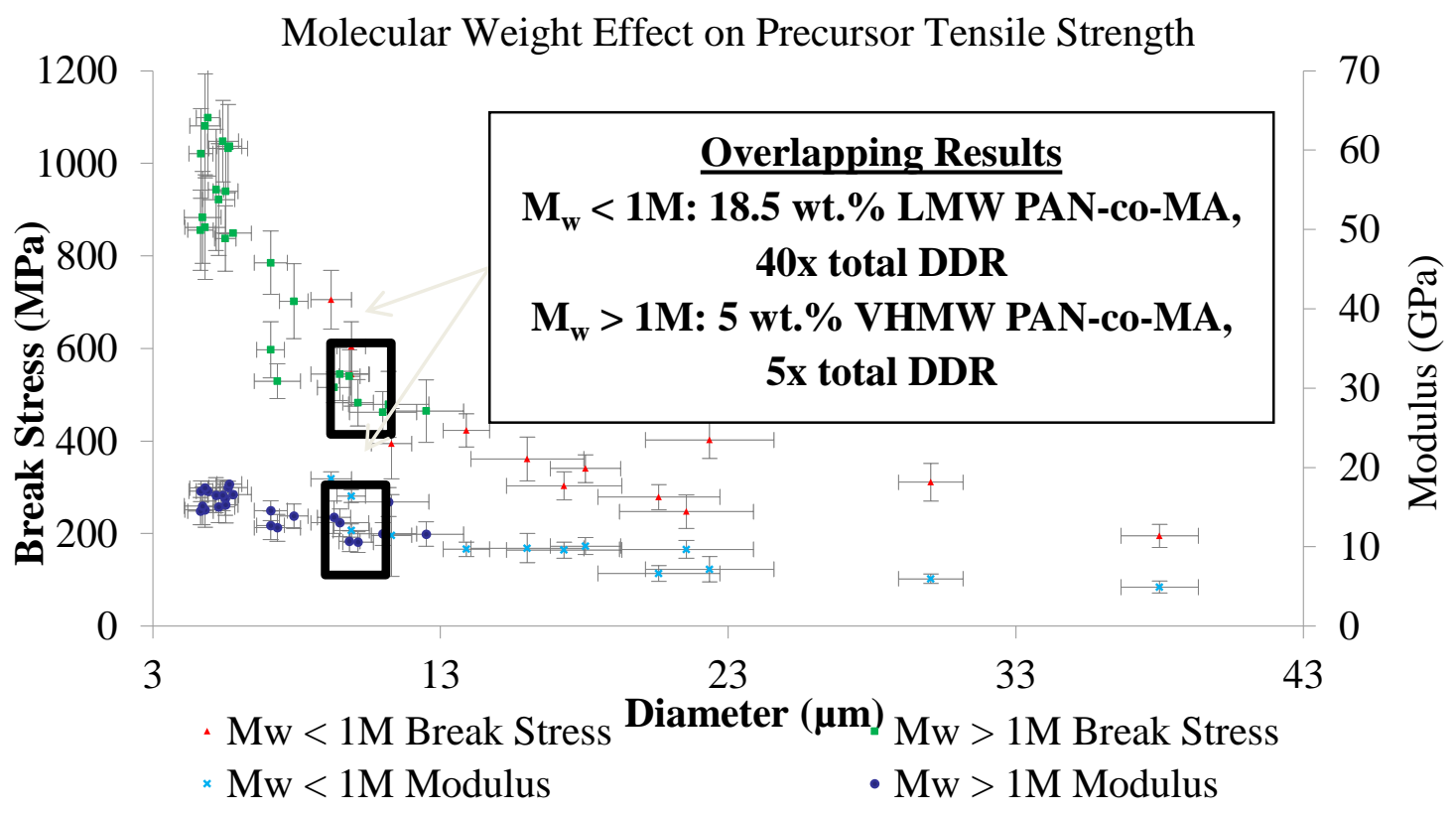

Figure 19. Results shown in Figure 17 separated into high vs. low molecular weight to show the impact of molecular weight on tensile strength 
If higher molecular weight polymers can be synthesized, lower solids content can be utilized to produce spinnable dopes. For example, modifying the following equation derived from the work of Fox (46),

$$
\eta_{0}=K V_{f} M_{w}^{3.4}
$$

where $\eta_{0}$ is polymer solution viscosity based on volume fraction of solids in the solution $\left(V_{f}\right)$, molecular weight $\left(M_{w}\right)$ of the polymer, and $\mathrm{K}$ is a constant related to polymer, solvent, and temperature (46), allows for extrapolation of the molecular weight necessary for spinnability at low dope polymer concentration (assuming equivalent solution viscosities).

Based on the above equation and experimental results for spinnable polymer solutions, it was extrapolated that a spinnable solution could be produced which required only $1 \mathrm{wt} \%$ solids content at a polymer molecular weight of 2.4 million. Neglecting the effects of die swell and stretch ratio, a change in dope solids content from $6 \mathrm{wt} \%$ to $1 \mathrm{wt} \%$ would result in approximately a $245 \%$ decrease in fiber diameter (from $37 \mu \mathrm{m}$ to $15 \mu \mathrm{m}$ ). The fiber produced in this study from a $6 \mathrm{wt} \%$ solids content resulted in a $5.1 \mu \mathrm{m}$ fiber diameter, with a tensile strength of $1 \mathrm{GPa}$, after a DDR $=43$. Assuming the decrease in diameter with decrease in solids content to $1 \mathrm{wt} \%$ is conserved as the fiber is stretched down the line at the same DDR, the resulting fiber would have an approximate diameter of $2.1 \mu \mathrm{m}$. Applying the power law relationship for tensile strength via fiber diameter derived in Figure $17\left(\mathrm{R}^{2}=0.91\right)$, a precursor filament $2.1 \mu \mathrm{m}$ in diameter would have a tensile strength of approximately $1.8 \mathrm{GPa}$. This is a conservative estimate, as the increase in molecular weight to $2.4 \mathrm{M}$ would likely allow for a DDR higher than 43 .

Ultimately, this work has shown that the use of VHMW polymers to achieve low solids content in the spinning solution, coupled with the increased drawability of VHMW polymers, led to smaller precursor fiber diameters, and as a result, higher tensile performance of the precursor fiber.

\section{Conclusions}

Synthesis of VHMW polyacrylonitrile-co-methyl acrylate copolymers with weight average molecular weights of at least 1.7 million $\mathrm{g} /$ mole were repeatedly achieved on a laboratory scale using emulsion polymerization, which is achievable in large scale commercial processes. SEC analysis of the VHMW samples in solvent with and without salt to break up aggregation identified the presence of polymer aggregates that are hypothesized to be associated with properties related to the processing of the spinning solution. Future efforts to further characterize the aggregation will be pursued at Virginia Tech.

Difficulties coagulating VHMW polymer-derived nascent filaments were overcome utilizing a hybrid dry-jet gel spinning technique. This hybrid dry-jet gel spinning technique utilized the solvent exchange process common in dry-jet solution spinning, while also utilizing a temperature-induced phase shift towards a gel fiber with tenacity capable of withstanding subsequent processing. Continuous, 100 filament count tows, 100s of meters in length were spun, analyzed, and their fiber form and mechanical properties were characterized. A power law relationship was identified between fiber diameter and fiber break stress and modulus. Experimentally, the hybrid dry-jet gel spinning method, coupled with VHMW polymers, produced precursor fibers with excellent tensile properties, averaging $954 \mathrm{MPa}$ in strength and 15.9 $\mathrm{GPa}$ in elastic modulus $(\mathrm{N}=296)$. There was a marked improvement in the ability of 
VHMW polymers to reach small filament diameters $(\sim 5 \mu \mathrm{m})$ compared to published data on commercial fibers. The higher molecular weight and lower solids content in the dope enabled high drawing and formation of small diameter filaments. In turn, these small diameter fibers exhibited higher tensile strength and modulus compared to those produced by conventional methods $(35,41,42,43)$. Extrapolation of the power law relationship between fiber diameter and tensile strength shows that a trend toward further increases in precursor tensile strength and modulus should be possible with further increases in precursor polymer molecular weight.

\section{Acknowledgements}

The authors would like to acknowledge financial support from ORNL and DARPA. Additionally, the authors would like to acknowledge ORNL researchers Dr. Felix Paulauskas, Dr. Amit Naskar, Dr. Soydan Ozcan, and Mr. Cliff Eberle for their support.

Funding for much of this work was provided by Oak Ridge National Laboratory U.S. Department of Energy under award number 400095449 and 4000100727 . NSF-MRI grant award number 1126534 at Virginia Tech provided equipment for molecular weight analysis. NSFDMR grant award number 1006630 was very helpful for much of the characterization and some of the synthesis.

\section{References}

1. Mathur RB, Bahl OP, and Mittal J. Composites Science and Technology 1993;51:223230.

2. Chen J, Wang C, Ge H, Bai Y, and Wang Y. J. Polym. Res. 2007;14(3):223-228.

3. Masson JC. Acrylic Fiber Technology and Applications. New York, NY: Marcel Dekker, Inc., 1995.

4. $\quad$ Peng GQ, Wen YF, Yang YG, and Liu L. Int. J. Polym. Anal. Charact. 2008;13:369-375.

5. Rahman MA, Ismail AF, Mustafa A, Ng BC, Hasbullah H, Rahaman MSA, and Abdullah MS. Development in Polymer-Based Carbon Fibre Research in Malaysia:169-179.

6. Maslowski E and Urbanska A. America's Textiles International 1989;18(FW2).

7. Morgan P. Carbon Fibers and Their Composites. Boca Raton, LA: Taylor \& Francis Group, 2005.

8. Chung DDL. Carbon Fiber Composites. Newton: Butterworth-Heinemann, 1994.

9. Odian G. Principles of Polymerization, 4th ed. Staten Island: Wiley-Interscience, 2004.

10. Kwon YD, Kavesh S, and Prevorsek DC. Patent 4,883,628. 1989.

11. Tsai JS and Lin CH. J. Appl. Polym. Sci. 1991;42:3045-3050.

12. Volokhina AV. Fibre Chemistry 2002;34(1):1-9.

13. Morris A and Weisenberger M. Tech Facts-Precursor Spinning for Carbon Fiber. 2013; Available from: http://www.caer.uky.edu/techfacts/TechFacts-CM-Precursor-SpinningUpdated-3June2013.pdf.

14. Baojun Q, Ding P, and Zhenqiou W. Adv. Polym. Technol. 1986;6(4):509-529.

15. Bajaj P, Sreekumar TV, and Sen K. J. Appl. Polym. Sci. 2002;86:773-787.

16. Sen K, Bahrami SH, and Bajaj P. Journal of Macromolecular Science Polymer Reviews 1996;36(1):1-76.

17. Konas M, Moy TM, Rogers ME, Shultz AR, Ward TC, and McGrath JE. Journal of Polymer Science Part B: Polymer Physics 1995;33(10):1429-1439. 
18. Jiang H, Yin Q, Zhou M, and Pan D. Adv. Mat. Res. 2012;430-432:687-691.

19. Kang JS and Lee YM. J. Appl. Polym. Sci. 2001;85(1).

20. Zhang X, Liu T, Sreekumar TV, Kumar S, Hu X, and Smith K. Polymer 2004;45:88018807.

21. $\quad$ Smith P and Lemstra PJ. J. Mater. Sci. 1980;15:505-514.

22. Sawai D, Fujii Y, and Kanamoto T. Polymer 2006;47:4445-4453.

23. Tsai JS and Su WC. J. Mater. Sci. Lett. 1991;10:1253-1256.

24. Wang YX, Wang CG, and Yu MJ. J. Appl. Polym. Sci. 2006;104:3723-3729.

25. Knudsen J. Text. Res. J. 1963;33(1):13-20.

26. Zeng X, Chen J, Zhao J, Wu C, Pan D, and Pan N. J. Appl. Polym. Sci. 2009;114:36213625.

27. Liu S, Tan L, Pan D, and Chen T. Polym. Int. 2010;60:453-457.

28. Widjojo N and Chung TS. Industrial and Engineering Chemistry Research 2006;45:76187626.

29. $\quad$ Chung TS and Hu X. J. Appl. Polym. Sci. 1997;66:1067-1077.

30. Tsai HA, Kuo CY, Lin JH, Wang DM, Deratani A, Pochat-Bohatier C, Lee KR, and Lai JY. J. Membr. Sci. 2006;278:390-400.

31. Morris A. Bench-Scale, Multifilament Spinning Conditions Effect on the Structure and Properties of Polyacrylonitrile Precursor Fiber. Master's Thesis. Lexington: University of Kentucky; 2011

32. Nohara LB, Filho GP, Nohara EL, Kleinke MU, and Rezende MC. Materials Research 2005;8(3):281-286.

33. Wang QF, Wang CG, Yu M, Ma J, Hu XY, and Zhu B. Science in China, Series E Technological Sciences 2010;53(6):1489-1494.

34. Leon y Leon CA. Patent 2084314 A2. 2009.

35. Kobashi T and Takao S. Patent 4,535,027. 1985.

36. Rahaman MSA, Ismail AF, and Mustafa A. Polym. Degrad. Stab. 2007;92:1421-1432.

37. Damodaran S, Desai P, and Abhiraman AS. Journal of the Textile Institute 1990;81(4):384-420.

38. Henrici-Olive G and Olive S. Adv. Polym. Sci. 1983;51:1-60.

39. Gupta AK, Paliwal DK, and Bajaj P. J. Macromol. Sci., Part B: Polym. Rev. 1991;C31(1):1-89.

40. Farsani RE. Production of Carbon Fibers from Acrylic Fibers. International Conference on Chemical, Civil, and Environment Engineering. Dubai, 2012. pp. 310-312.

41. Matsuhisa Y, Kibayashi M, Yamasaki K, and Okuda A. Patent 6,438,892 B2. 2002.

42. Wangxi Z, Jie L, and Gang W. Carbon 2003;41:2805-2812.

43. Ohsaki T, Imai K, and Miyahara N. Patent 4,925,604. 1990.

44. Griffith AA. Philos. Trans. R. Soc. London, A 1921;221:163-198.

45. Sawai D, Yamane A, Takahashi H, Kanamoto T, Ito M, and Porter RS. J. Polym. Sci., Part B: Polym. Phys. 1998;36:629-640.

46. Fox TG and Flory PJ. Journal of Physical and Colloid Chemistry 1951;55(221). 


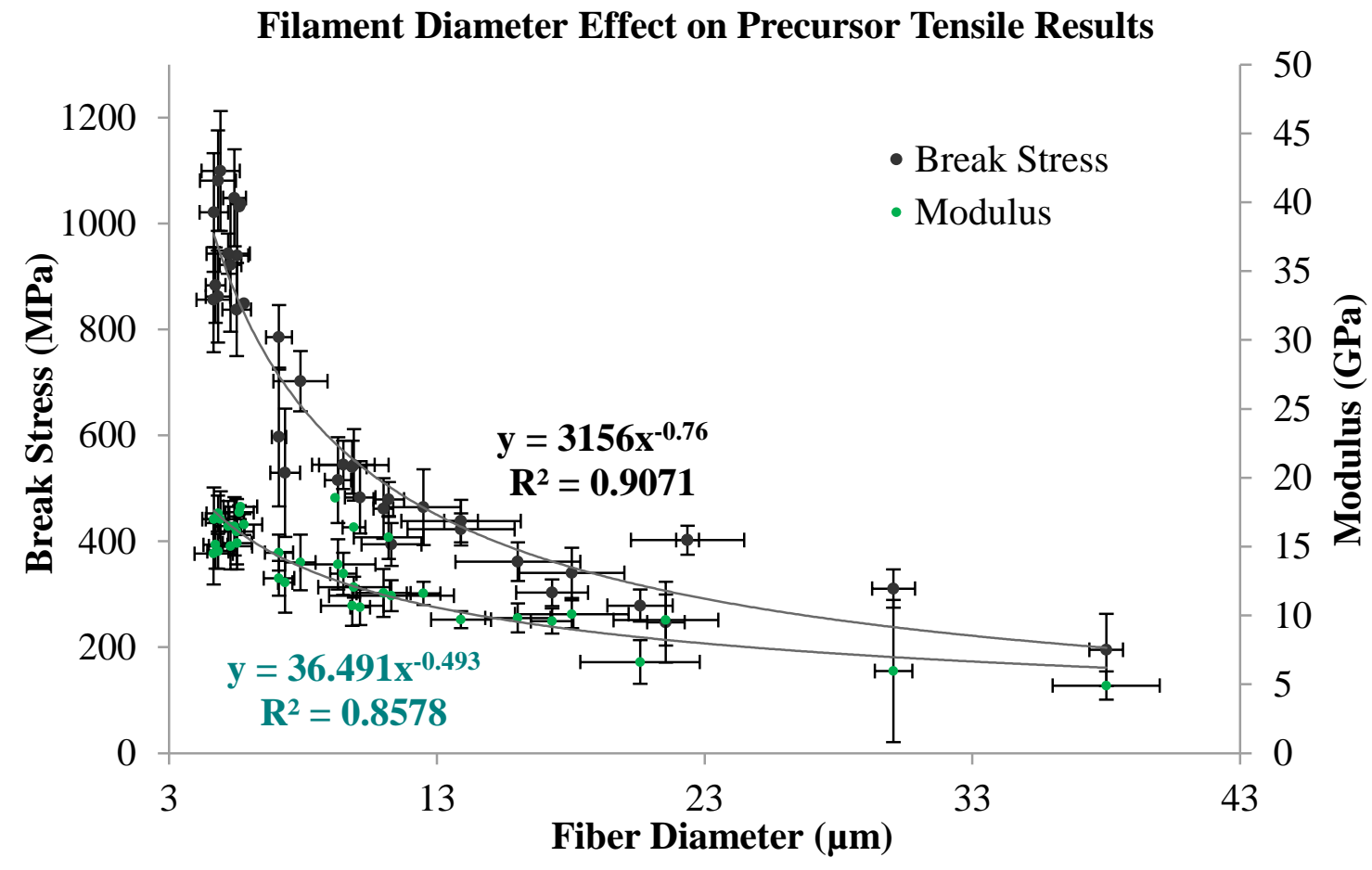

